\title{
METRICALLY REGULAR MAPPING AND ITS UTILIZATION TO CONVERGENCE ANALYSIS OF A RESTRICTED INEXACT NEWTON-TYPE METHOD*
}

\author{
Mohammed Harunor Rashid \\ Institute of Computational Mathematics and Scientific/Engineering Computing, Academy of \\ Mathematics and Systems Science, Chinese Academy of Sciences, Beijing 100190, China; \\ Department of Mathematics, Faculty of Science, University of Rajshahi, Rajshahi-6205, Bangladesh \\ Email: harun_math@ru.ac.bd
}

\begin{abstract}
In the present paper, we study the restricted inexact Newton-type method for solving the generalized equation $0 \in f(x)+F(x)$, where $X$ and $Y$ are Banach spaces, $f: X \rightarrow Y$ is a Fréchet differentiable function and $F: X \rightrightarrows Y$ is a set-valued mapping with closed graph. We establish the convergence criteria of the restricted inexact Newton-type method, which guarantees the existence of any sequence generated by this method and show this generated sequence is convergent linearly and quadratically according to the particular assumptions on the Fréchet derivative of $f$. Indeed, we obtain semilocal and local convergence results of restricted inexact Newton-type method for solving the above generalized equation when the Fréchet derivative of $f$ is continuous and Lipschitz continuous as well as $f+F$ is metrically regular. An application of this method to variational inequality is given. In addition, a numerical experiment is given which illustrates the theoretical result.
\end{abstract}

Mathematics subject classification: 47H04, 49J53, 65K10, 90C30.

Key words: Generalized equation, Restricted inexact Newton-type method, Metrically regular mapping, Partial Lipschitz-like mapping, Semilocal convergence.

\section{Introduction}

Let $X$ and $Y$ be Banach spaces, $f: X \rightarrow Y$ be a Fréchet differentiable function, and $F: X \rightrightarrows Y$ be a set-valued mapping with closed graph. In this paper, we are intended to find a point that satisfies the following generalized equation

$$
0 \in f(x)+F(x) .
$$

The generalized equations of the type (1.1) were introduced by Robinson [48]. This type of generalized equation problem is an abstract model for a wide variety of variational problems including linear and nonlinear complementarity problems, systems of nonlinear equations, systems of inequalities and variational inequalities (see $[48,50]$ for more details). In particular, it may characterize optimality or equilibrium problems (see $[24,28]$ for more details).

The classical Newton-type method is one of the most important method for finding an approximate solution of (1.1), which was introduced by Dontchev [16] and defined as follows:

$$
0 \in f\left(x_{k}\right)+\mathcal{D} f\left(x_{k}\right)\left(x_{k+1}-x_{k}\right)+F\left(x_{k+1}\right), \quad \text { for } k=0,1, \ldots,
$$

\footnotetext{
${ }^{*}$ Received January 30, 2019 / Revised version received June 13, 2019 / Accepted May 19, 2020 /

Published online September 24, 2020 /
} 
where $x_{0}$ is a given initial point and $\mathcal{D} f(x)$ is the derivative of $f$ at $x$. When $F \equiv 0$, the above method reduces to the standard Newton method for solving the equation $f(x)=0$ of the form:

$$
f\left(x_{k}\right)+\mathcal{D} f\left(x_{k}\right)\left(x_{k+1}-x_{k}\right)=0, \quad \text { for } k=0,1, \ldots
$$

There is a vast biographical research on inexact Newton-type methods for solving equation $f(x)=0$ which employs different representation of inexactness; see for examples $[7,8,54]$.

For solving generalized equation (1.1), Klatte and Kumer [32] generated a Newton sequence whose Newton steps are defined by approximations $f^{(k)}$ of $f$ near the current iterate $x_{k}$ and the solutions $x_{k+1}$ of

$$
0 \in f^{(k)}(x)+F(x),
$$

and concentrated on local convergence analysis for Newton's method under certain type of approximations and different regularity conditions for $f+F$. Moreover, the authors [32, Proposition 8] have presented a Kantorovich-type statement, which is concerned on semilocal convergence, under pseudo-regularity of $f+F$ provided that all constructed Newton sequences are valid. Moreover, Aragon Artacho et al. [4] introduced Newton's iteration and presented its convergence analysis under metrically regular mapping when the single-valued part of the generalized equation (1.1) is an implicit function with a parameter. For solving generalized equation (1.1), a survey of local and semilocal convergence results for Newton's method can be found in $[3,5,9,16,18,19,24,37]$ and references therein.

In the case when the involved single-valued function $f$ is not necessarily differentiable, we say that the generalized equation (1.1) is nonsmooth. The authors in [1] introduced a mapping $\mathcal{H}: X \rightrightarrows \mathcal{L}(X, Y)$ and applied a selection $\psi: X \rightarrow \mathcal{L}(X, Y)$ for $\mathcal{H}$ to the following method for solving nonsmooth generalized equation (1.1) and obtained a superlinear convergent result:

$$
0 \in f\left(x_{k}\right)+\mathcal{H}\left(x_{k}\right)\left(x_{k+1}-x_{k}\right)+F\left(x_{k+1}\right) \quad \text { for } k=0,1,2, \ldots
$$

For solving nonsmooth generalized equation (1.1), Cibulka et al. [13] studied an inexact Newton method and obtained local convergence results for the method. Moreover, the semi-smooth Newton-type iterative procedure, for solving (1.1), was adopted by Cibulka et al. [14] (also see $[24$, Section $6 \mathrm{~F}]$ ). An extension of [33, Lemma 10.1] from equation to nonsmooth model (1.1) is given in [32, Theorem 4] via the concept of Newton maps [33]. Relevant results, for solving nonsmooth generalized equations (1.1), are given in $[2,6,19,27,38,39,49,52]$.

A large number of new developments on Newton methods with regularity properties of setvalued mappings for solving nonsmooth generalized equations have been studied in the last three decades and some of which have been accumulated in the monographs $[17,31,33,53]$.

Dembo et al. [15] introduced the following inexact Newton method for solving (1.1) with $F \equiv 0, X=Y=\mathbb{R}^{n}$ and $f$ continuously differentiable with Jacobian $\nabla f$ in finite dimensional case:

$$
\left(f\left(x_{k}\right)+\nabla f\left(x_{k}\right)\left(x_{k+1}-x_{k}\right)\right) \cap \mathbb{B}\left(0, \eta_{k}\left\|f\left(x_{k}\right)\right\|\right) \neq \emptyset,
$$

where $\left\{\eta_{k}\right\} \subset(0, \infty)$ is a sequence of scalars and $\mathbb{B}(x, \alpha)$ denotes the closed ball centered at $x$ with radius $\alpha$.

Izmailov and Solodov [30] (see also in the monograph [31]) introduced the following inexact Newton method for solving the generalized equation (1.1) in the case of finite dimension:

$$
0 \in f\left(x_{k}\right)+\nabla f\left(x_{k}\right)\left(x_{k+1}-x_{k}\right)+e_{k}+F\left(x_{k+1}\right), \quad \text { where } e_{k} \in \mathbb{R}^{n} .
$$


In order to represent the inexactness for the method (1.2), Dontchev and Rockafellar [25] associated the following inexact Newton method for solving the generalized equation (1.1) in the Banach space setting:

$$
\left(f\left(x_{k}\right)+\mathcal{D} f\left(x_{k}\right)\left(x_{k+1}-x_{k}\right)+F\left(x_{k+1}\right)\right) \cap \mathcal{R}_{k}\left(x_{k}, x_{k+1}\right) \neq \emptyset, \quad \text { for } k=0,1, \ldots,
$$

where $\mathcal{R}_{k}: X \times X \rightrightarrows Y$ is a sequence of set-valued mappings with closed graph which represents the inexactness for the general model (1.2). When the starting point is sufficiently close to the solution, under the metric regularity properties of the mapping $f+F$ and $\mathcal{R}_{k}(\cdot, \cdot)$, it has been shown in [25] that every sequence generated by the method (1.5) is convergent either q-linearly, q-superlinearly or q-quadratically according to the particular assumptions. It has also been noted in [25] that when $F \equiv 0$ and $\mathcal{R}_{k}\left(x_{k}, x_{k+1}\right)=\mathbb{B}\left(0, \eta_{k}\left\|f\left(x_{k}\right)\right\|\right)$, (1.5) coincides with the iterative method (1.4). An exact and inexact Humel-Seebeck type methods for approximating the solution of (1.1) were proposed and studied by Burnet, Jean-Alexis and Piétrus [12]. To solve the nonsmooth generalized equation (1.1), Cibulka et al. [13] associated the following inexact Newton methods

$$
\left(f\left(x_{k}\right)+A_{k}\left(x_{k}\right)\left(x_{k+1}-x_{k}\right)+F\left(x_{k+1}\right)\right) \cap \mathcal{R}_{k}\left(x_{k}\right) \neq \emptyset, \quad \text { for } k=0,1, \ldots,
$$

where $\mathcal{H}: X \rightrightarrows \mathcal{L}(X, Y)$ is a generalized set-valued derivative of $f$ with $A_{k} \in \mathcal{H}\left(x_{k}\right)$ and the mapping $\mathcal{R}_{k}: X \rightrightarrows Y$ represents inexactness which depends on the current iteration $x_{k}$ only. Under the assumptions of metric regularity properties of the mapping $f+F$, local convergence results of exact and inexact Newton methods are obtained by many investigators (see e.g. [24, Section $6 \mathrm{C} \& \mathrm{E}]$ and $[1,4,26,32]$ respectively).

Let $x \in X$. The subset of $X$, denoted by $\Lambda_{\triangle}(x)$, is defined by

$$
\Lambda_{\triangle}(x):=\left\{s \in X:(f(x)+\mathcal{D} f(x) s+F(x+s)) \cap \mathcal{R}_{x}(x, x+s) \neq \emptyset\right\} .
$$

Usually, for a starting point near to the solution, theorems in $[13,25,30,31]$ are focused on the existence of one sequence, which is convergent to the solution of (1.1). In particular, under certain conditions the convergence result, established in [25, Theorem 4], guarantees the existence of one sequence $\left\{x_{k}\right\}$, which is linearly convergent to the solution. Hence, from a numerical point of view, the iteration scheme (1.5) is not convenient in practical application. This drawback motivates us to propose the following restricted inexact Newton-type method (see Algorithm 1.1).

Algorithm 1.1. (The Restricted Inexact Newton-type method(RINM))

Step 1. Let $\eta \in[1, \infty)$ and given $x_{0} \in X$, and put $k:=0$.

Step 2. If $0 \in \Lambda_{\triangle}\left(x_{k}\right)$ stop; otherwise go to Step 3.

Step 3. If $0 \notin \Lambda_{\triangle}\left(x_{k}\right)$, choose $s_{k}$ such that $s_{k} \in \Lambda_{\triangle}\left(x_{k}\right)$ and

$$
\left\|s_{k}\right\| \leq \eta \mathrm{d}\left(0, \Lambda_{\triangle}\left(x_{k}\right)\right) .
$$

Step 4. Update by $x_{k+1}:=x_{k}+s_{k}$.

Step 5. Update by $k:=k+1$ and go to Step 2 .

Note that in comparison to the method (1.5), it sees that when $\eta=1$ and the set $\Lambda_{\triangle}\left(x_{k}\right)$ is singleton for each $k=0,1,2, \ldots$, the method (1.5) and Algorithm 1.1 are coincident; otherwise 
Algorithm 1.1 is a restricted version of the iteration scheme (1.5) because it involves a restricted term $\left\|s_{k}\right\| \leq \eta \mathrm{d}\left(0, \Lambda_{\triangle}\left(x_{k}\right)\right)$ on the length of $s_{k}$. Moreover, in contrast with the contribution in [25] it seems that the local convergence results of the method (1.5) are presented by the authors in [25] while in our current paper we present the semilocal convergence of the restricted Newtontype method. For solving (1.1), different version of exact and inexact iterative methods have been introduced and studied their local and semilocal convergence analysis in $[1,4,12,13,41-46]$ and the references therein.

In this paper, we analyze semilocal convergence of restricted Newton-type method for solving (1.1). Metrically regular property of a set-valued mapping is the key tool in our investigation. This property has been studied by many mathematicians; see, for example, [9, 17, 21, 22, 29, 44] and the references therein. Based on the information around the initial point, our main results are the convergence criteria, presented in Section 3, which provide some sufficient conditions assuring the convergence to a solution of any sequence generated by Algorithm 1.1. As a result, local convergence results of the restricted Newton-type method are obtained.

The rest of this paper is organized as follows. In the next section, we give some necessary notations and recall some preliminary results. The main theorems are presented in Section 3 , where the semilocal and local convergence results of the sequence generated by Algorithm 1.1 for metrically regular mapping are established. In Section 4, we provide an application of RINM to variational inequality and present a numerical experiment to illustrate the theoretical results. Summary of the major results, presented in this paper, are mentioned in the last section.

\section{Notations and Preliminary Results}

In this section we recall some standard notations and notions. Let $X, Y$ and $Q$ be Banach spaces. Let $x \in X$ and $\alpha \in(0, \infty)$. We use $\mathbb{B}(x, \alpha)$ to denote the closed ball centered at $x$ with radius $\alpha$. The set of all natural numbers is denote by $\mathbb{N}$ and $\mathbb{N}_{0}=\mathbb{N} \cup\{0\}$. When a sequence of positive scalars $\left\{w_{k}\right\}$ is convergent to zero, we write $w_{k} \searrow 0$. Let $A \subseteq X$ and $C \subseteq X$. The distance from $x$ to $A$ is defined by

$$
\mathrm{d}(x, A):=\inf _{a \in A}\|x-a\|,
$$

while the excess from the set $A$ to the set $C$ is defined by

$$
e(C, A):=\sup _{c \in C} \mathrm{~d}(c, A)
$$

Let $\Gamma: X \rightrightarrows Y$ be a set-valued mapping. The domain dom $\Gamma$, the graph gph $\Gamma$ and the inverse $\Gamma^{-1}$ of $\Gamma$ are respectively defined by

$$
\begin{gathered}
\operatorname{dom} \Gamma:=\{x \in X: \Gamma(x) \neq \emptyset\} ; \operatorname{gph} \Gamma:=\{(x, y) \in X \times Y: y \in \Gamma(x)\} \\
\text { and } \Gamma^{-1}(y):=\{x \in X: y \in \Gamma(x)\}
\end{gathered}
$$

Recall the notions of the metric regularities for a set-valued mapping in the following definition from [44], which have been studied extensively; see for example [23, 24, 34, 36, 46, 51].

Definition 2.1. Let $T: X \rightrightarrows Y$ be a set-valued mapping, and let $(\bar{x}, \bar{y}) \in \operatorname{gph} T$. Let $r_{\bar{x}}>$ $0, r_{\bar{y}}>0$ and $\kappa>0$. Then $T$ is said to be 
(i) metrically regular at $(\bar{x}, \bar{y})$ on $\mathbb{B}\left(\bar{x}, r_{\bar{x}}\right) \times \mathbb{B}\left(\bar{y}, r_{\bar{y}}\right)$ with constant $\kappa$ if

$$
\mathrm{d}\left(x, T^{-1}(y)\right) \leq \kappa \mathrm{d}(y, T(x)) \quad \text { for all } x \in \mathbb{B}\left(\bar{x}, r_{\bar{x}}\right), y \in \mathbb{B}\left(\bar{y}, r_{\bar{y}}\right) .
$$

(ii) metrically regular at $(\bar{x}, \bar{y})$ if there exist constants $r_{\bar{x}}^{\prime}>0, r_{\bar{y}}^{\prime}>0$ and $\kappa^{\prime}>0$ such that $T$ is metrically regular at $(\bar{x}, \bar{y})$ on $\mathbb{B}\left(\bar{x}, r_{\bar{x}}^{\prime}\right) \times \mathbb{B}\left(\bar{y}, r_{\bar{y}}^{\prime}\right)$ with constant $\kappa^{\prime}$.

The infimum of the set of values $\kappa$ for which (2.1) holds with some $r_{\bar{x}}>0, r_{\bar{y}}>0$ is called the modulus of metric regularity, denoted by $\operatorname{reg}(T ;(\bar{x}, \bar{y}))$; see [23] and the references therein for more details.

The notions of pseudo-Lipschitz and Lipschitz-like properties for set-valued mapping are extracted from [47]. These notions have been introduced by Aubin [10,11] and studied extensively. For their connections between linear rate of openness, coderivatives, metric regularity of setvalued mappings and applications to variational problems, one can refer to $[23,34,36]$ and the monographs [35, 51].

Definition 2.2. Let $\Gamma: X \rightrightarrows Y$ be a set-valued mapping and let $(\bar{x}, \bar{y}) \in$ gph $\Gamma$. Let $r_{\bar{x}}>$ $0, r_{\bar{y}}>0$ and $M>0$. Then $\Gamma$ is said to be

(i) Lipchitz-like at $(\bar{x}, \bar{y})$ on $\mathbb{B}\left(\bar{x}, r_{\bar{x}}\right) \times \mathbb{B}\left(\bar{y}, r_{\bar{y}}\right)$ with constant $M$ if

$$
e\left(\Gamma\left(x_{1}\right) \cap \mathbb{B}\left(\bar{y}, r_{\bar{y}}\right), \Gamma\left(x_{2}\right)\right) \leq M\left\|x_{1}-x_{2}\right\| \quad \text { for any } \quad x_{1}, x_{2} \in \mathbb{B}\left(\bar{x}, r_{\bar{x}}\right) .
$$

(ii) pseudo-Lipchitz around $(\bar{x}, \bar{y})$ if there exist constants $r_{\bar{x}}^{\prime}>0, r_{\bar{y}}^{\prime}>0$ and $M^{\prime}>0$ such that $\Gamma$ is Lipschitz-like at $(\bar{x}, \bar{y})$ on $\mathbb{B}\left(\bar{x}, r_{\bar{x}}^{\prime}\right) \times \mathbb{B}\left(\bar{y}, r_{\bar{y}}^{\prime}\right)$ with constant $M^{\prime}$.

Remark 2.1. Equivalently, for the property (i) in Definition 2.2, we can say that $\Gamma$ is Lipschitzlike at $(\bar{x}, \bar{y}) \in \operatorname{gph} \Gamma$ on $\mathbb{B}\left(\bar{x}, r_{\bar{x}}\right) \times \mathbb{B}\left(\bar{y}, r_{\bar{y}}\right)$ with constant $M$ if for every $y_{1}, y_{2} \in \mathbb{B}\left(\bar{y}, r_{\bar{y}}\right)$ and for every $y_{1} \in \Gamma\left(x_{1}\right) \cap \mathbb{B}\left(\bar{y}, r_{\bar{y}}\right)$, there exists $y_{2} \in \Gamma\left(x_{2}\right)$ such that

$$
\left\|y_{1}-y_{2}\right\| \leq M\left\|x_{1}-x_{2}\right\|, \quad \text { for every } x_{1}, x_{2} \in \mathbb{B}\left(\bar{x}, r_{\bar{x}}\right)
$$

We employ the following definitions of partial Lipschitz-like and partially pseudo-Lipschitz properties for set-valued mapping of two variables from [25].

Definition 2.3. Let $S: Q \times X \rightrightarrows Y$ be a set-valued mapping with $((\bar{q}, \bar{x}), \bar{y}) \in \operatorname{gph} S$. Let $r_{\bar{q}}>0, r_{\bar{x}}>0, r_{\bar{y}}>0$ and $L>0$. Then $S$ is said to be

(i) partially Lipschitz-like at $((\bar{q}, \bar{x}), \bar{y})$ with respect to $x$ on $\left(\mathbb{B}\left(\bar{q}, r_{\bar{p}}\right) \times \mathbb{B}\left(\bar{x}, r_{\bar{x}}\right)\right) \times \mathbb{B}\left(\bar{y}, r_{\bar{y}}\right)$ with constant $L$ if, for any $x, x^{\prime} \in \mathbb{B}\left(\bar{x}, r_{\bar{x}}\right)$ and $q \in \mathbb{B}\left(\bar{q}, r_{\bar{q}}\right)$, the following condition holds

$$
e\left(S(q, x) \cap \mathbb{B}\left(\bar{y}, r_{\bar{y}}\right), S\left(q, x^{\prime}\right)\right) \leq L\left\|x-x^{\prime}\right\| .
$$

(ii) partially pseudo-Lipschitz around $((\bar{q}, \bar{x}), \bar{y})$ if there exist constants $r_{\bar{q}}^{\prime}>0, r_{\bar{x}}^{\prime}>0, r_{\bar{y}}^{\prime}>$ 0 and $L^{\prime}>0$ such that $S$ is partially Lipschitz-like at $((\bar{q}, \bar{x}), \bar{y})$ with respect to $x$ on $\left(\mathbb{B}\left(\bar{q}, r_{\bar{p}}^{\prime}\right) \times \mathbb{B}\left(\bar{x}, r_{\bar{x}}^{\prime}\right)\right) \times \mathbb{B}\left(\bar{y}, r_{\bar{y}}^{\prime}\right)$ with constant $L^{\prime}$.

The proof of the following lemma is given in [44] or in [24]. This lemma establishes the connection between the metric regularity and the Lipchitz-like property, which will be useful. After combining this lemma with [40, Remark 2.1], we state the modified form as follows: 
Lemma 2.1. Let $T: X \rightrightarrows Y$ be a set-valued mapping and let $(\bar{x}, \bar{y}) \in \operatorname{gph} T$. Assume that $T$ is metrically regular at $(\bar{x}, \bar{y})$ on $\mathbb{B}\left(\bar{x}, r_{\bar{x}}\right) \times \mathbb{B}\left(\bar{y}, r_{\bar{y}}\right)$ with constant $\kappa$. Then for every $y, y^{\prime} \in \mathbb{B}\left(\bar{y}, r_{\bar{y}}\right)$ and for any $x_{1} \in T^{-1}(y) \cap \mathbb{B}\left(\bar{x}, r_{\bar{x}}\right)$, there exists $x_{2} \in T^{-1}\left(y^{\prime}\right)$ such that

$$
\left\|x_{1}-x_{2}\right\| \leq \kappa\left\|y-y^{\prime}\right\| .
$$

Recall the following statement which is a refinement of the Lyusternik-Graves theorem for metrically regular mapping taken from [21, Theorem 3.3]. Analogue developments on this result appear in [17, Theorem 1.4] or Section 1 in Ioffe [29]. This theorem plays an important role in the theory of metric regularity. This theorem proves the stability of metric regularity of a set-valued mapping for generalized equation under perturbations.

Lemma 2.2. Let $T: X \rightrightarrows Y$ be a set-valued mapping and let $(\bar{x}, \bar{y}) \in \operatorname{gph} T$. Let $T$ be a metrically regular at $(\bar{x}, \bar{y})$ on $\mathbb{B}\left(\bar{x}, r_{\bar{x}}\right) \times \mathbb{B}\left(\bar{y}, r_{\bar{y}}\right)$ with constant $\kappa>0$ and $\operatorname{gph} T \cap\left(\mathbb{B}\left(\bar{x}, r_{\bar{x}}\right) \times\right.$ $\left.\mathbb{B}\left(\bar{y}, r_{\bar{y}}\right)\right)$ be closed. Suppose that $g: X \rightarrow Y$ is a Lipschitz continuous function with Lipschitz constant $\lambda$ such that $\lambda \kappa<1$. Then the mapping $g+T$ is metrically regular at $(\bar{x}, \bar{y}+g(\bar{x}))$ on $\mathbb{B}\left(\bar{x}, r_{\bar{x}}\right) \times \mathbb{B}\left(\bar{y}+g(\bar{x}), r_{\bar{y}}\right)$ with constant $\frac{\kappa}{1-\kappa \lambda}$.

We finish this section with the following coincidence theorem, which is taken from [25, Theorem 1] and it was originally proved in [20].

Lemma 2.3. Let $X$ and $Y$ be two metric spaces. Suppose that $\Phi: X \rightrightarrows Y$ and $\theta: Y \rightrightarrows X$ are set-valued mappings. Let $r \in(0,+\infty), \kappa>0, \lambda>0$ and $\bar{x} \in X, \bar{y} \in Y$ be such that $\kappa \lambda<1$. Assume that one of the sets $\operatorname{gph} \Phi \cap\left(\mathbb{B}(\bar{x}, r) \times \mathbb{B}\left(\bar{y}, \frac{r}{\lambda}\right)\right)$ and $\operatorname{gph} \theta \cap\left(\mathbb{B}\left(\bar{y}, \frac{r}{\lambda}\right) \times \mathbb{B}(\bar{x}, r)\right)$ is closed while the other is complete, or both sets $\operatorname{gph}(\Phi \cdot \theta) \cap\left(\mathbb{B}\left(\bar{y}, \frac{r}{\lambda}\right) \times \mathbb{B}\left(\bar{y}, \frac{r}{\lambda}\right)\right)$ and $\operatorname{gph}(\theta \cdot \Phi) \cap(\mathbb{B}(\bar{x}, r) \times \mathbb{B}(\bar{x}, r))$ are complete. Also, suppose that the following conditions hold:

$$
\begin{aligned}
& \mathrm{d}(\bar{y}, \Phi(\bar{x}))<r(1-\kappa \lambda) /(2 \lambda) ; \\
& \mathrm{d}(\bar{x}, \theta(\bar{y}))<r(1-\kappa \lambda) / 2 ; \\
& e\left(\Phi\left(x_{1}\right) \cap \mathbb{B}\left(\bar{y}, \frac{r}{\lambda}\right), \Phi\left(x_{2}\right)\right) \leq \kappa\left\|x_{1}-x_{2}\right\|, \text { for all } x_{1}, x_{2} \in \mathbb{B}(\bar{x}, r) ;
\end{aligned}
$$

and

$$
e\left(\theta\left(y_{1}\right) \cap \mathbb{B}(\bar{x}, r), \theta\left(y_{2}\right)\right) \leq \lambda\left\|y_{1}-y_{2}\right\|, \text { for all } y_{1}, y_{2} \in \mathbb{B}\left(\bar{y}, \frac{r}{\lambda}\right) .
$$

Then there exist $\hat{x} \in \mathbb{B}(\bar{x}, r)$ and $\hat{y} \in \mathbb{B}\left(\bar{y}, \frac{r}{\lambda}\right)$ such that $\hat{y} \in \Phi(\hat{x})$ and $\hat{x} \in \theta(\hat{y})$. Additionally, if $\Phi$ and $\theta$ are single-valued, then $\hat{y}=\Phi(\hat{x})$ and $\hat{x}=\theta(\hat{y})$ for $\hat{x} \in \mathbb{B}(\bar{x}, r)$ and $\hat{y} \in \mathbb{B}\left(\bar{y}, \frac{r}{\lambda}\right)$.

\section{Convergence Analysis}

Throughout this section, we suppose that $X$ and $Y$ are Banach spaces. Let $f: X \rightarrow Y$ be a Fréchet differentiable function and its derivative is denoted by $\mathcal{D} f$. Let $\mathcal{R}_{x}: X \times X \rightrightarrows Y$ and $F: X \rightrightarrows Y$ be set-valued mappings with closed graph. Let $r_{\bar{x}}>0$ and $r_{\bar{y}}>0$. Moreover, in this section we consider the restricted inexact Newton-type method(RINM) defined by Algorithm 1.1 for solving the generalized equation (1.1). Let $x \in X$. For the simplicity, define a set-valued mapping $\mathcal{W}_{x}: X \rightrightarrows Y$ by

$$
\mathcal{W}_{x}(\cdot):=f(x)+\mathcal{D} f(x)(\cdot-x)+F(\cdot)
$$


Thus, by the definition of $\Lambda_{\triangle}(x)$, we obtain that

$$
\Lambda_{\triangle}(x)=\left\{s \in X: \mathcal{W}_{x}(x+s) \cap \mathcal{R}_{x}(x, x+s) \neq \emptyset\right\} .
$$

Moreover, one can easily see that the following equivalence is trivial:

$$
u \in \mathcal{W}_{x}^{-1}(w) \Leftrightarrow w \in f(x)+\mathcal{D} f(x)(u-x)+F(u) \text { for any } u \in X \text { and } w \in Y .
$$

In particular, for every $(\bar{x}, \bar{y}) \in \operatorname{gph}(f+F)$, we have that

$$
\bar{x} \in \mathcal{W}_{\bar{x}}^{-1}(\bar{y})
$$

Now, let $x, u \in X$ and $w \in Y$. Then, by the formation of $\mathcal{W}_{x}$ in (3.1) together with (3.3) we obtain that

$$
w \in \mathcal{W}_{x}(u)=f(x)+\mathcal{D} f(x)(u-x)+F(u)
$$

which can be written as

$$
w-f(x)-\mathcal{D} f(x)(u-x) \in F(u) .
$$

This, together with the definition of $\mathcal{W}_{u}$, implies that

$$
\mathcal{W}_{u}(u)=f(u)+F(u) \ni f(u)+w-f(x)-\mathcal{D} f(x)(u-x) .
$$

Put

$$
Z(x, u):=f(x)+\mathcal{D} f(x)(u-x)-f(u) .
$$

Then, (3.5) implies that

$$
w-Z(x, u) \in \mathcal{W}_{u}(u)
$$

When the approximation of $f$ can be represented by a classical linearization, we have the following lemma which is due to [46]:

Lemma 3.1. Let $f: X \rightarrow Y$ be a Fréchet differentiable function on $\mathbb{B}\left(\bar{x}, r_{\bar{x}}\right)$ and suppose that the set-valued mapping $F: X \rightrightarrows Y$ is metrically regular at $(\bar{x}, \bar{y}-f(\bar{x}))$ on $\mathbb{B}\left(\bar{x}, r_{\bar{x}}\right) \times$ $\mathbb{B}\left(\bar{y}-f(\bar{x}), r_{\bar{y}}\right)$ with constant $\kappa>0$, which have locally closed graph at $(\bar{x}, \bar{y}-f(\bar{x}))$ with $\bar{y} \in f(\bar{x})+F(\bar{x})$. Then the following statements are equivalent:

(i) The mapping $f+F$ is metrically regular at $(\bar{x}, \bar{y})$ on $\mathbb{B}\left(\bar{x}, r_{\bar{x}}\right) \times \mathbb{B}\left(\bar{y}, r_{\bar{y}}\right)$ with constant $\kappa$.

(ii) The mapping $\mathcal{W}_{\bar{x}}$ is metrically regular at $(\bar{x}, \bar{y})$ on $\mathbb{B}\left(\bar{x}, r_{\bar{x}}\right) \times \mathbb{B}\left(\bar{y}, r_{\bar{y}}\right)$ with constant $\kappa$.

Let $\varepsilon>0$ and define

$$
b:=\min \left\{r_{\bar{y}}-2 \varepsilon r_{\bar{x}}, \frac{r_{\bar{x}}(1-\kappa \varepsilon)}{4 \kappa}\right\} .
$$

Then

$$
b>0 \Longleftrightarrow \varepsilon<\min \left\{\frac{r_{\bar{y}}}{2 r_{\bar{x}}}, \frac{1}{\kappa}\right\} .
$$

Recall the following lemma from [46, Lemma 3.2]. This lemma plays an important role for convergence analysis of RINM. 
Lemma 3.2. Assume that the mapping $\mathcal{W}_{\bar{x}}$ is metrically regular at $(\bar{x}, \bar{y})$ on $\mathbb{B}\left(\bar{x}, r_{\bar{x}}\right) \times \mathbb{B}\left(\bar{y}, r_{\bar{y}}\right)$ with constant $\kappa$. Let $b$ be defined in (3.8) so that (3.9) is satisfied. Let $x \in \mathbb{B}\left(\bar{x}, \frac{r_{\bar{x}}}{2}\right)$ and assume that $\mathcal{D} f$ is continuous on $\mathbb{B}\left(\bar{x}, \frac{r_{\bar{x}}}{2}\right)$ with constant $\varepsilon$. Then $\mathcal{W}_{x}$ is metrically regular at $(\bar{x}, \bar{y})$ on $\mathbb{B}\left(\bar{x}, \frac{r_{\bar{x}}}{2}\right) \times \mathbb{B}(\bar{y}, b)$ with constant $\frac{\kappa}{1-\kappa \varepsilon}$, that is, for any $u_{1} \in \mathbb{B}\left(\bar{x}, \frac{r_{\bar{x}}}{2}\right), v_{2} \in \mathbb{B}(\bar{y}, b)$,

$$
\mathrm{d}\left(u_{1}, \mathcal{W}_{x}^{-1}\left(v_{2}\right)\right) \leq \frac{\kappa}{1-\kappa \varepsilon} \mathrm{d}\left(v_{2}, \mathcal{W}_{x}\left(u_{1}\right)\right)
$$

Before going to present our first main result, for each $x \in X$ and $y \in Y$, we define a function $h_{x}: X \rightarrow Y$ by

$$
h_{x}(\cdot):=f(\bar{x})+\mathcal{D} f(\bar{x})(\cdot-\bar{x})-f(x)-\mathcal{D} f(x)(\cdot-x),
$$

set-valued mappings $\Xi_{x}: X \rightrightarrows Y$ by

$$
\Xi_{x}(\cdot)=\mathcal{R}_{x}(x, \cdot)
$$

and $\Upsilon: Y \rightrightarrows X$ by

$$
\Upsilon_{x}(\cdot)=\mathcal{W}_{x}^{-1}(\cdot)
$$

Then, for every $u^{\prime}, u^{\prime \prime} \in X$, we have

$$
\left\|h_{x}\left(u^{\prime}\right)-h_{x}\left(u^{\prime \prime}\right)\right\| \leq\|\mathcal{D} f(\bar{x})-\mathcal{D} f(x)\|\left\|u^{\prime}-u^{\prime \prime}\right\| .
$$

\subsection{Linear Convergence}

In this subsection, we assume that $\mathcal{W}_{\bar{x}}$ is metrically regular at $(\bar{x}, \bar{y})$ on $\mathbb{B}\left(\bar{x}, r_{\bar{x}}\right) \times \mathbb{B}\left(\bar{y}, r_{\bar{y}}\right)$ with constant $\kappa>0$ and $\operatorname{gph} W_{\bar{x}} \cap\left(\mathbb{B}\left(\bar{x}, r_{\bar{x}}\right) \times \mathbb{B}\left(\bar{y}, r_{\bar{y}}\right)\right)$ is closed. Further, assume that $\mathcal{R}_{x}(\cdot, \cdot)$ is partially Lipschitz-like at $((\bar{x}, \bar{x}), \bar{y})$ on $\left(\mathbb{B}\left(\bar{x}, r_{\bar{x}}\right) \times \mathbb{B}\left(\bar{x}, r_{\bar{x}}\right)\right) \times \mathbb{B}\left(\bar{y}, r_{\bar{y}}\right)$ with constant $\mu$. We study here the semi-local and local convergence of the sequence generated by Algorithm 1.1 with initial point $x_{0}$ when $\mathcal{W}_{\bar{x}}$ is metrically regular at $(\bar{x}, \bar{y})$ on $\mathbb{B}\left(\bar{x}, r_{\bar{x}}\right) \times \mathbb{B}\left(\bar{y}, r_{\bar{y}}\right)$.

Theorem 3.1. Let $\eta>1$ and suppose that $\mathcal{W}_{\bar{x}}$ is metrically regular at $(\bar{x}, \bar{y})$ on $\mathbb{B}\left(\bar{x}, r_{\bar{x}}\right) \times$ $\mathbb{B}\left(\bar{y}, r_{\bar{y}}\right)$ with constant $\kappa>0$ and $\operatorname{gph} \mathcal{W}_{\bar{x}} \cap\left(\mathbb{B}\left(\bar{x}, r_{\bar{x}}\right) \times \mathbb{B}\left(\bar{y}, r_{\bar{y}}\right)\right)$ is closed. Let $x \in \mathbb{B}\left(\bar{x}, \frac{r_{\bar{x}}}{2}\right)$ and suppose that $\mathcal{D} f$ is continuous on $\mathbb{B}\left(\bar{x}, \frac{r_{\bar{x}}}{2}\right)$ with constant $\varepsilon$ and let $b$ be defined in (3.8) so that $b>0$. Let $u \in X$ and assume that $\mathcal{R}_{x}(x, u)$ is partially Lipschitz-like at $((\bar{x}, \bar{x}), \bar{y})$ on $\left(\mathbb{B}\left(\bar{x}, \frac{r_{\bar{x}}}{2}\right) \times \mathbb{B}\left(\bar{x}, \frac{r_{\bar{x}}}{2}\right)\right) \times \mathbb{B}(\bar{y}, b)$ with constant $\mu>0$ so that $\varepsilon(4 \eta+1) \kappa<1-\kappa \mu$. Let $\delta>0$ be such that

$$
\delta \leq \min \left\{\frac{r_{\bar{x}}}{4}, \frac{b}{3 \varepsilon}, 1, \frac{b \kappa}{1-\kappa \varepsilon}\right\} \text { and }\|\bar{y}\|<\varepsilon \delta .
$$

Suppose that

$$
\lim _{x \rightarrow \bar{x}} \mathrm{~d}\left(\bar{y}, \mathcal{W}_{x}(x)\right)=0 \text { and } \mathrm{d}\left(\bar{y}, \mathcal{R}_{x}(x, \bar{x})\right) \leq \varepsilon\|x-\bar{x}\| .
$$

Then, for every $k \in \mathbb{N}_{0}$ and every $w_{k} \in \mathbb{B}(\bar{y}, b)$ with $w_{k} \in \mathcal{R}_{x}(x, u)$ satisfying

$$
\mathcal{W}_{x}(u) \ni w_{k}
$$

there exists some $\hat{\delta}>0$ such that for any initial point $x_{0} \in \mathbb{B}(\bar{x}, \hat{\delta})$, Algorithm 1.1 generates a sequence, which may not be unique, and any generated sequence $\left\{x_{k}\right\}$ converges linearly to a solution $x^{*} \in \mathbb{B}\left(\bar{x}, r_{\bar{x}}\right)$ of $(1.1)$. 
Proof. The mapping $\mathcal{W}_{\bar{x}}$ is metrically regular at $(\bar{x}, \bar{y})$ on $\mathbb{B}\left(r_{\bar{x}}, \bar{x}\right) \times \mathbb{B}\left(r_{\bar{y}}, \bar{y}\right)$ with constant $\kappa$. Then through Lemma $3.2, \mathcal{W}_{x}$ is metrically regular at $(\bar{x}, \bar{y})$ on $\mathbb{B}\left(\bar{x}, \frac{r_{\bar{x}}}{2}\right) \times \mathbb{B}(\bar{y}, b)$ with constant $\lambda:=\frac{\kappa}{1-\kappa \varepsilon}$, that is,

$$
\mathrm{d}\left(u_{1}, \mathcal{W}_{x}^{-1}\left(v_{2}\right)\right) \leq \lambda \mathrm{d}\left(v_{2}, \mathcal{W}_{x}\left(u_{1}\right)\right) \text { for any } u_{1} \in \mathbb{B}\left(\bar{x}, \frac{r_{\bar{x}}}{2}\right), v_{2} \in \mathbb{B}(\bar{y}, b) .
$$

It is obvious that $\kappa(\mu+\varepsilon)<1$, since the condition $\varepsilon(4 \eta+1) \kappa<1-\kappa \mu$ holds. Since $\lambda=\frac{\kappa}{1-\kappa \varepsilon}$, it follows that $\lambda \mu<1$. The continuity property of $\mathcal{D} f$ on $\mathbb{B}\left(\bar{x}, \frac{r_{\bar{x}}}{2}\right)$ with constant $\varepsilon$ yields that

$$
\left\|\mathcal{D} f(x)-\mathcal{D} f\left(x^{\prime}\right)\right\| \leq \varepsilon, \quad \text { for all } x, x^{\prime} \in \mathbb{B}\left(\bar{x}, \frac{r_{\bar{x}}}{2}\right)
$$

Then, we obtain, for all $u^{\prime}, v^{\prime} \in \mathbb{B}\left(\bar{x}, \frac{r_{\bar{x}}}{2}\right)$, that

$$
\begin{aligned}
& \left\|f\left(u^{\prime}\right)-f\left(v^{\prime}\right)-\mathcal{D} f\left(v^{\prime}\right)\left(u^{\prime}-v^{\prime}\right)\right\| \\
= & \left\|\int_{0}^{1} \mathcal{D} f\left(v^{\prime}+t\left(u^{\prime}-v^{\prime}\right)\right)\left(u^{\prime}-v^{\prime}\right) d t-\mathcal{D} f\left(v^{\prime}\right)\left(u^{\prime}-v^{\prime}\right)\right\| \\
\leq & \int_{0}^{1}\left\|\mathcal{D} f\left(v^{\prime}+t\left(u^{\prime}-v^{\prime}\right)\right)-\mathcal{D} f\left(v^{\prime}\right)\right\|\left\|u^{\prime}-v^{\prime}\right\| d t \\
\leq & \varepsilon\left\|u^{\prime}-v^{\prime}\right\| \int_{0}^{1} d t=\varepsilon\left\|u^{\prime}-v^{\prime}\right\| .
\end{aligned}
$$

Moreover, the partial Lipschitz-like property of $\mathcal{R}_{x}(x, u)$ at $((\bar{x}, \bar{x}), \bar{y})$ on $\left(\mathbb{B}\left(\bar{x}, \frac{r_{\bar{x}}}{2}\right) \times \mathbb{B}\left(\bar{x}, \frac{r_{\bar{x}}}{2}\right)\right) \times$ $\mathbb{B}(\bar{y}, b)$ with constant $\mu$ imply, for all $x, x^{\prime}, x^{\prime \prime} \in \mathbb{B}\left(\bar{x}, \frac{r_{\bar{x}}}{2}\right)$, that

$$
e\left(\mathcal{R}_{x}\left(x, x^{\prime}\right) \cap \mathbb{B}(\bar{y}, b), \mathcal{R}_{x}\left(x, x^{\prime \prime}\right)\right) \leq \mu\left\|x^{\prime}-x^{\prime \prime}\right\|
$$

Let $x_{0} \in \mathbb{B}(\hat{\delta}, \bar{x})$ and $w_{n} \in \mathbb{B}(\bar{y}, b)$ for $n=0,1, \ldots$. Since the first condition in (3.15) and (3.16) are hold, we can infer $0<\hat{\delta} \leq \delta$ for which the following inequality holds:

$$
\mathrm{d}\left(w_{0}, \mathcal{W}_{x_{0}}\left(x_{0}\right)\right)<\varepsilon \delta, \quad \text { for each } x_{0} \in \mathbb{B}(\bar{x}, \hat{\delta}) \text { and } w_{0} \in \mathbb{B}(\bar{y}, b)
$$

Set

$$
l:=\eta \lambda \varepsilon=\frac{\eta \kappa \varepsilon}{1-\kappa \varepsilon} .
$$

Moreover, due to $\varepsilon(4 \eta+1) \kappa<1-\kappa \mu$, we obtain that

$$
\frac{4 \eta \kappa \varepsilon}{1-\kappa \varepsilon} \leq \frac{4 \eta \kappa \varepsilon}{1-\kappa(\varepsilon+\mu)}<1 \text {. }
$$

It follows, from (3.22), that

$$
l<\frac{1}{4}
$$

To complete the proof, it is sufficient to show that the Algorithm 1.1 generates at least one sequence and any generated sequence $\left\{x_{n}\right\}$ satisfies

$$
\left\|s_{n}\right\| \leq l^{n+1} \delta
$$

and

$$
\left(x_{n+1}, w_{n}\right) \in \operatorname{gph} \mathcal{W}_{x_{n}} \cap\left(\mathbb{B}\left(\bar{x}, \frac{r_{\bar{x}}}{2}\right) \times \mathbb{B}(\bar{y}, b)\right)
$$


for each $n=0,1,2, \ldots$ The proof will be proceed by induction on $n$. For this connection, we define, for each $x \in X$,

$$
\beta_{x}:=\frac{2 \lambda \varepsilon}{1-\lambda \mu}\|x-\bar{x}\| .
$$

Moreover, since $\eta>1$, the inequality $\varepsilon(4 \eta+1) \kappa<1-\kappa \mu$ gives that

$$
\frac{2 \lambda \varepsilon}{1-\lambda \mu}=\frac{2 \kappa \varepsilon}{1-\kappa(\varepsilon+\mu)} \leq \frac{2 \eta \kappa \varepsilon}{1-\kappa(\varepsilon+\mu)}<\frac{1}{2}
$$

It follows from $(3.26)$, for each $x \in \mathbb{B}(\bar{x}, 2 \delta)$, that

$$
\beta_{x} \leq \frac{4 \lambda \varepsilon \delta}{1-\lambda \mu}<\delta
$$

First of all, we will prove that

$$
\Lambda_{\triangle}\left(x_{0}\right) \neq \emptyset,
$$

which will ensure the existence of the point $x_{1}$. To do this, we consider the mappings $\Xi_{x_{0}}$ and $\Upsilon_{x_{0}}$ defined by (3.11) and (3.12) respectively. Now, we show that assumptions (2.4), (2.5), (2.6) and (2.7) of Lemma 2.3 are hold with $\kappa:=\lambda, \lambda:=\mu, \bar{x}:=\bar{x}, \bar{y}:=\bar{y}$ and $c:=\beta_{x_{0}}$. Granting this, Lemma 2.3 is applicable to conclude that there exist fixed points $\hat{x} \in \mathbb{B}\left(\bar{x}, \beta_{x_{0}}\right)$ and $\hat{y} \in \mathbb{B}\left(\bar{y}, \frac{\beta_{x_{0}}}{\lambda}\right)$ such that

$$
\hat{y} \in \Xi_{x_{0}}(\hat{x}) \text { and } \hat{x} \in \Upsilon_{x_{0}}(\hat{y}) .
$$

The inclusion $\hat{x} \in \Upsilon_{x_{0}}(\hat{y})$ translates to $\hat{y} \in \mathcal{W}_{x_{0}}(\hat{x})$, that is, $\hat{y} \in f\left(x_{0}\right)+\mathcal{D} f\left(x_{0}\right)\left(\hat{x}-x_{0}\right)+F(\hat{x})$. This, together with the inclusion $\hat{y} \in \Xi_{x_{0}}(\hat{x})=\mathcal{R}_{x_{0}}\left(x_{0}, \hat{x}\right)$ by (3.11) and (3.29), implies that

$$
\hat{y} \in\left(f\left(x_{0}\right)+\mathcal{D} f\left(x_{0}\right)\left(\hat{x}-x_{0}\right)+F(\hat{x})\right) \cap \mathcal{R}_{x_{0}}\left(x_{0}, \hat{x}\right)
$$

and therefore, (3.28) is hold.

Let us check that assumptions (2.4), (2.5), (2.6) and (2.7) of Lemma 2.3 are hold for the mappings $\Phi:=\Xi_{x_{0}}$ and $\theta:=\Upsilon_{x_{0}}$ with $\kappa:=\lambda, \lambda:=\mu, \bar{x}:=\bar{x}, \bar{y}:=\bar{y}$ and $c:=\beta_{x_{0}}$.

The second condition of (3.15) gives, for $x_{0} \in \mathbb{B}\left(\bar{x}, \beta_{x_{0}}\right) \subseteq \mathbb{B}(\bar{x}, \delta) \subseteq \mathbb{B}\left(\bar{x}, \frac{r_{\bar{x}}}{2}\right)$, that

$$
\mathrm{d}\left(\bar{y}, \mathcal{R}_{x_{0}}\left(x_{0}, \bar{x}\right)\right) \leq \varepsilon\left\|x_{0}-\bar{x}\right\| .
$$

It follows from (3.11) that

$$
\begin{aligned}
\mathrm{d}\left(\bar{y}, \Xi_{x_{0}}(\bar{x})\right) & =\mathrm{d}\left(\bar{y}, \mathcal{R}_{x_{0}}\left(x_{0}, \bar{x}\right)\right) \leq \varepsilon\left\|x_{0}-\bar{x}\right\| \\
& \leq \frac{\beta_{x_{0}}(1-\lambda \mu)}{2 \lambda}=: \frac{c(1-\lambda \mu)}{2 \lambda} .
\end{aligned}
$$

This implies that the assertion (2.4) of Lemma 2.3 is hold.

Moreover, using (3.19) and third inequality from (3.14), we obtain, for any $x \in \mathbb{B}(\bar{x}, 2 \delta)$, that

$$
\begin{aligned}
& \left\|\left(\bar{y}-h_{x_{0}}(x)\right)-\bar{y}\right\| \\
= & \left\|\bar{y}-f(\bar{x})-\mathcal{D} f(\bar{x})(x-\bar{x})+f\left(x_{0}\right)+\mathcal{D} f\left(x_{0}\right)\left(x-x_{0}\right)-\bar{y}\right\| \\
\leq & \left\|f(\bar{x})-f\left(x_{0}\right)-\mathcal{D} f\left(x_{0}\right)\left(\bar{x}-x_{0}\right)\right\|+\left\|\mathcal{D} f\left(x_{0}\right)-\mathcal{D} f(\bar{x})\right\|\|\bar{x}-x\| \\
\leq & \varepsilon\left(\left\|\bar{x}-x_{0}\right\|+\|\bar{x}-x\|\right) \leq 3 \varepsilon \delta \leq b .
\end{aligned}
$$


Letting $x=\bar{x}$ in (3.31) and this gives that

$$
\left\|\left(\bar{y}-h_{x_{0}}(\bar{x})\right)-\bar{y}\right\| \leq \varepsilon\left\|\bar{x}-x_{0}\right\| \leq \varepsilon \delta \leq b .
$$

The above relations show that $\bar{y}-h_{x_{0}}(x) \in \mathbb{B}(\bar{y}, b)$ for all $x \in \mathbb{B}(\bar{x}, 2 \delta)$. Hence from the formation of $\mathcal{W}_{x}$ in (3.1), we have

$$
\bar{y}-h_{x_{0}}(\bar{x}) \in \mathcal{W}_{x_{0}}(\bar{x}) \cap \mathbb{B}(\bar{y}, b) .
$$

Thus, by virtue of the metric regularity of $\mathcal{W}_{x_{0}}$ from (3.17), definition of $\Upsilon$ in (3.12) and the construction of $h_{x_{0}}$, we obtain, for $x_{0} \in \mathbb{B}\left(\bar{x}, \beta_{x_{0}}\right) \subseteq \mathbb{B}(\bar{x}, \delta) \subseteq \mathbb{B}\left(\bar{x}, \frac{r_{\bar{x}}}{2}\right)$, that

$$
\mathrm{d}\left(\bar{x}, \Upsilon_{x_{0}}(\bar{y})\right)=\mathrm{d}\left(\bar{x}, \mathcal{W}_{x_{0}}^{-1}(\bar{y})\right) \leq \lambda \mathrm{d}\left(\bar{y}, \mathcal{W}_{x_{0}}(\bar{x})\right) \leq \lambda\left\|\left(\bar{y}-h_{x_{0}}(\bar{x})\right)-\bar{y}\right\| .
$$

This together with (3.32) gives that

$$
\mathrm{d}\left(\bar{x}, \Upsilon_{x_{0}}(\bar{y})\right) \leq \lambda\left\|\left(\bar{y}-h_{x_{0}}(\bar{x})\right)-\bar{y}\right\| \leq \lambda \varepsilon\left\|\bar{x}-x_{0}\right\|=\frac{1}{2} \beta_{x_{0}}(1-\lambda \mu):=\frac{c}{2}(1-\lambda \mu) .
$$

Hence, assertion (2.5) of Lemma 2.3 is hold.

To show assumptions (2.6) and (2.7) of Lemma 2.3 are hold, let $x^{\prime}, x^{\prime \prime} \in \mathbb{B}\left(\bar{x}, \beta_{x_{0}}\right)$. Then by the relation $4 \delta \leq r_{\bar{x}}$ in (3.14) and (3.27), we have $x^{\prime}, x^{\prime \prime} \in \mathbb{B}\left(\bar{x}, \beta_{x_{0}}\right) \subseteq \mathbb{B}(\bar{x}, \delta) \subseteq \mathbb{B}\left(\bar{x}, \frac{r_{\bar{x}}}{2}\right)$. Moreover, by (3.27) and (3.14) we have that

$$
\beta_{x_{0}} \leq \delta \leq \lambda b \Rightarrow \frac{\beta_{x_{0}}}{\lambda} \leq b .
$$

For any $x^{\prime}, x^{\prime \prime} \in \mathbb{B}\left(\bar{x}, \beta_{x_{0}}\right)$, we have from (3.20) that

$$
\begin{aligned}
& e\left(\Xi_{x_{0}}\left(x^{\prime}\right) \cap \mathbb{B}\left(\bar{y}, \frac{\beta_{x_{0}}}{\lambda}\right), \Xi_{x_{0}}\left(x^{\prime \prime}\right)\right) \\
\leq & e\left(\Xi_{x_{0}}\left(x^{\prime}\right) \cap \mathbb{B}(\bar{y}, b), \Xi_{x_{0}}\left(x^{\prime \prime}\right)\right) \\
= & e\left(\mathcal{R}_{x_{0}}\left(x_{0}, x^{\prime}\right) \cap \mathbb{B}(\bar{y}, b), \mathcal{R}_{x_{0}}\left(x_{0}, x^{\prime \prime}\right)\right) \\
\leq & \mu\left\|x^{\prime}-x^{\prime \prime}\right\| .
\end{aligned}
$$

This shows that the assertion (2.6) of Lemma 2.3 is hold.

Finally, we show that the assertion (2.7) of Lemma 2.3 is also hold. To show this, let $y, y^{\prime} \in \mathbb{B}\left(\bar{y}, \frac{\beta_{x_{0}}}{\lambda}\right)$. Let $u_{1} \in \mathcal{W}_{x_{0}}^{-1}(y) \cap \mathbb{B}\left(\bar{x}, \beta_{x_{0}}\right)$. Since $\mathcal{W}_{x_{0}}$ is metrically regular at $(\bar{x}, \bar{y})$ on $\mathbb{B}\left(\bar{x}, \frac{r_{\bar{x}}}{2}\right) \times \mathbb{B}(\bar{y}, b)$ with constant $\lambda$, by $(3.17)$ we have that

$$
\mathrm{d}\left(u_{1}, \mathcal{W}_{x_{0}}^{-1}\left(y^{\prime}\right)\right) \leq \lambda \mathrm{d}\left(y^{\prime}, \mathcal{W}_{x_{0}}\left(u_{1}\right)\right) \leq \lambda\left\|y-y^{\prime}\right\| .
$$

By the definition of excess $e$, we have that

$$
\begin{aligned}
& e\left(\Upsilon_{x_{0}}(y) \cap \mathbb{B}\left(\bar{x}, \beta_{x_{0}}\right), \Upsilon_{x_{0}}\left(y^{\prime}\right)\right) \\
\leq & e\left(\mathcal{W}_{x_{0}}^{-1}(y) \cap \mathbb{B}\left(\bar{x}, \frac{r_{\bar{x}}}{2}\right), \mathcal{W}_{x_{0}}^{-1}\left(y^{\prime}\right)\right) \\
= & \sup \left\{\mathrm{d}\left(u_{1}, \mathcal{W}_{x_{0}}^{-1}\left(y^{\prime}\right)\right): u_{1} \in \mathcal{W}_{x_{0}}^{-1}(y) \cap \mathbb{B}\left(\bar{x}, \frac{r_{\bar{x}}}{2}\right)\right\} .
\end{aligned}
$$

This, together with (3.35), gives that

$$
e\left(\Upsilon_{x_{0}}(y) \cap \mathbb{B}\left(\bar{x}, \beta_{x_{0}}\right), \Upsilon_{x_{0}}\left(y^{\prime}\right)\right) \leq \lambda\left\|y-y^{\prime}\right\| .
$$


It follows that assumption (2.7) of Lemma 2.3 is satisfied. Hence, (3.28) is established, and consequently, we can choose $s_{0}$ such that $s_{0} \in \Lambda_{\triangle}\left(x_{0}\right)$ and

$$
\left\|s_{0}\right\| \leq \eta \mathrm{d}\left(0, \Lambda_{\triangle}\left(x_{0}\right)\right)
$$

Therefore, by Algorithm 1.1, $x_{1}:=x_{0}+s_{0}$ is defined. By the choice of $s_{0}$, we have from (3.2) that

$$
\Lambda_{\triangle}\left(x_{0}\right):=\left\{s_{0} \in X: \mathcal{W}_{x_{0}}\left(x_{0}+s_{0}\right) \cap \mathcal{R}_{x_{0}}\left(x_{0}, x_{0}+s_{0}\right) \neq \emptyset\right\}
$$

Since for every $w_{0} \in \mathcal{R}_{x_{0}}\left(x_{0}, x_{0}+s_{0}\right),(3.16)$ is satisfied, then we have that $w_{0} \in \mathcal{W}_{x_{0}}\left(x_{0}+s_{0}\right) \Rightarrow$ $x_{0}+s_{0} \in \mathcal{W}_{x_{0}}^{-1}\left(w_{0}\right)$. Thus, it follows from (3.38) that

$$
\Lambda_{\triangle}\left(x_{0}\right)=\left\{s_{0} \in X: w_{0} \in \mathcal{W}_{x_{0}}\left(x_{0}+s_{0}\right)\right\}=\left\{s_{0} \in X: x_{0}+s_{0} \in \mathcal{W}_{x_{0}}^{-1}\left(w_{0}\right)\right\} .
$$

Moreover, taking into account (3.39), we obtain that

$$
\mathrm{d}\left(0, \Lambda_{\triangle}\left(x_{0}\right)\right)=\mathrm{d}\left(x_{0}, \mathcal{W}_{x_{0}}^{-1}\left(w_{0}\right)\right) .
$$

Applying the metrically regular property of $\mathcal{W}_{x_{0}}$ from (3.17), we get, from (3.37) and (3.40), that

$$
\left\|s_{0}\right\| \leq \eta \mathrm{d}\left(x_{0}, \mathcal{W}_{x_{0}}^{-1}\left(w_{0}\right)\right) \leq \eta \lambda \mathrm{d}\left(w_{0}, \mathcal{W}_{x_{0}}\left(x_{0}\right)\right) .
$$

This gives, for (3.21), that

$$
\left\|s_{0}\right\| \leq \eta \lambda \mathrm{d}\left(w_{0}, \mathcal{W}_{x_{0}}\left(x_{0}\right)\right) \leq \eta \lambda \varepsilon \delta=l \delta,
$$

which shows that (3.24) holds for $n=0$. Furthermore, (3.41) gives that

$$
\left\|s_{0}\right\|=\left\|x_{1}-x_{0}\right\| \leq l \delta<\delta .
$$

Consequently, we obtain that

$$
\left\|x_{1}-\bar{x}\right\| \leq\left\|x_{1}-x_{0}\right\|+\left\|x_{0}-\bar{x}\right\|<2 \delta .
$$

This implies that $x_{1} \in \mathbb{B}(\bar{x}, 2 \delta) \subseteq \mathbb{B}\left(\bar{x}, \frac{r_{\bar{x}}}{2}\right)$. Moreover, since $x_{1}=x_{0}+s_{0}$ is generated by Algorithm 1.1, (3.39) gives that

$$
\Lambda_{\triangle}\left(x_{0}\right)=\left\{s_{0} \in X: x_{0}+s_{0} \in \mathcal{W}_{x_{0}}^{-1}\left(w_{0}\right)\right\}=\left\{s_{0} \in X: x_{1} \in \mathcal{W}_{x_{0}}^{-1}\left(w_{0}\right)\right\} .
$$

This, together with $x_{1} \in \mathbb{B}\left(\bar{x}, \frac{r_{\bar{x}}}{2}\right)$ and $w_{0} \in \mathbb{B}(\bar{y}, b)$, implies that (3.25) holds for $n=0$.

We proceed by induction. Indeed, we are going to show that every sequence generated by Algorithm 1.1 satisfies (3.24) and (3.25) for all $n$. To do this, we assume that the points $x_{0}, x_{1}, \ldots, x_{k+1}$ are obtained by Algorithm 1.1 with initial point $x_{0}$ such that (3.24) and (3.25) hold for $n=0,1, \ldots, k-1$ and show that assertions (3.24) and (3.25) hold for $n=k$. Since (3.24) and (3.25) are true for $n=0,1, \ldots, k-1$, by Algorithm 1.1, $x_{k}:=x_{k-1}+s_{k-1}$ is defined and thus $x_{k} \in \mathbb{B}(\bar{x}, 2 \delta) \subseteq \mathbb{B}\left(\bar{x}, \frac{r_{\bar{x}}}{2}\right)$. In this stage, if we impose almost same arguments that used for the case of $n=0$, one can apply Lemma 2.3 to the mappings $\Phi:=\Xi_{x_{k}}$ and $\theta:=\Upsilon_{x_{k}}$ with $\kappa:=\lambda, \lambda:=\mu, \bar{x}:=\bar{x}, \bar{y}:=\bar{y}$ and $c:=\beta_{x_{k}}$ and show that $\Lambda_{\triangle}\left(x_{k}\right) \neq \emptyset$. Then, choose $s_{k}$ such that $s_{k} \in \Lambda_{\triangle}\left(x_{k}\right)$ and

$$
\left\|s_{k}\right\| \leq \eta \mathrm{d}\left(0, \Lambda_{\triangle}\left(x_{k}\right)\right)
$$


Applying Algorithm 1.1, we have that $x_{k+1}:=x_{k}+s_{k}$ is defined. By the choice of $s_{k}$, we obtain from (3.2) that

$$
\Lambda_{\triangle}\left(x_{k}\right):=\left\{s_{k} \in X: \mathcal{W}_{x_{k}}\left(x_{k}+s_{k}\right) \cap \mathcal{R}_{x_{k}}\left(x_{k}, x_{k}+s_{k}\right) \neq \emptyset\right\}
$$

For any $w_{k} \in \mathcal{R}_{x_{k}}\left(x_{k}, x_{k}+s_{k}\right)$, we obtain, through (3.16), that $w_{k} \in \mathcal{W}_{x_{k}}\left(x_{k}+s_{k}\right) \Rightarrow x_{k}+s_{k} \in$ $\mathcal{W}_{x_{k}}^{-1}\left(w_{k}\right)$. Since $\mathcal{W}_{x_{k}}\left(x_{k}+s_{k}\right) \cap \mathcal{R}_{x_{k}}\left(x_{k}, x_{k}+s_{k}\right) \neq \emptyset$ and $w_{k} \in \mathcal{W}_{x_{k}}\left(x_{k}+s_{k}\right) \cap \mathcal{R}_{x_{k}}\left(x_{k}, x_{k}+s_{k}\right)$, it follows from (3.43) that

$$
\begin{aligned}
\Lambda_{\triangle}\left(x_{k}\right) & =\left\{s_{k} \in X: w_{k} \in \mathcal{W}_{x_{k}}\left(x_{k}+s_{k}\right)\right\} \\
& =\left\{s_{k} \in X: x_{k}+s_{k} \in \mathcal{W}_{x_{k}}^{-1}\left(w_{k}\right)\right\} .
\end{aligned}
$$

From (3.44), we get

$$
\mathrm{d}\left(0, \Lambda_{\triangle}\left(x_{k}\right)\right)=\mathrm{d}\left(x_{k}, \mathcal{W}_{x_{k}}^{-1}\left(w_{k}\right)\right) .
$$

Moreover, for $x_{k-1}, x_{k} \in \mathbb{B}(\bar{x}, 2 \delta) \subseteq \mathbb{B}\left(\bar{x}, \frac{r_{\bar{x}}}{2}\right)$ and $w_{k} \in \mathbb{B}(\bar{y}, b)$, we have, from (3.6) and (3.7), that

$$
\left.w_{k}-Z\left(x_{k-1}, x_{k}\right) \in \mathcal{W}_{x_{k}}\left(x_{k}\right)\right) \text {. }
$$

Again, applying the metrically regular property of the mapping $\mathcal{W}_{x_{k}}$, we get from (3.42) that

$$
\begin{aligned}
\left\|s_{k}\right\| & \leq \eta \mathrm{d}\left(x_{k}, \mathcal{W}_{x_{k}}^{-1}\left(w_{k}\right)\right) \leq \eta \lambda \mathrm{d}\left(w_{k}, \mathcal{W}_{x_{k}}\left(x_{k}\right)\right) \\
& \leq \eta \lambda\left\|w_{k}-\left(w_{k}-Z\left(x_{k-1}, x_{k}\right)\right)\right\| \\
& \leq \eta \lambda\left\|f\left(x_{k}\right)-f\left(x_{k-1}\right)-\mathcal{D} f\left(x_{k-1}\right)\left(x_{k}-x_{k-1}\right)\right\| \\
& \leq \eta \lambda \varepsilon\left\|x_{k}-x_{k-1}\right\| \leq l \cdot l^{k} \delta=l^{k+1} \delta .
\end{aligned}
$$

Hence, (3.24) holds for each $n=k$. Using (3.45), we obtain

$$
\left\|x_{k+1}-\bar{x}\right\| \leq \sum_{i=0}^{k}\left\|s_{i}\right\|+\left\|x_{0}-\bar{x}\right\| \leq \delta \sum_{i=0}^{k} l^{i+1}+\delta \leq \frac{l \delta}{1-l}+\delta \leq 2 \delta,
$$

which shows that $x_{k+1} \in \mathbb{B}(\bar{x}, 2 \delta) \subseteq \mathbb{B}\left(\bar{x}, \frac{r_{\bar{x}}}{2}\right)$. On the other hand, Algorithm 1.1 generates the point $x_{k+1}$ such that $x_{k+1}=x_{k}+s_{k}$ is defined. Therefore, (3.44) yields that

$$
\Lambda_{\triangle}\left(x_{k}\right)=\left\{s_{k} \in X: x_{k}+s_{k} \in \mathcal{W}_{x_{k}}^{-1}\left(w_{k}\right)\right\}=\left\{s_{k} \in X: x_{k+1} \in \mathcal{W}_{x_{k}}^{-1}\left(w_{k}\right)\right\} .
$$

This, together with $x_{k+1} \in \mathbb{B}\left(\bar{x}, \frac{r_{\bar{x}}}{2}\right)$ and $w_{k} \in \mathbb{B}(\bar{y}, b)$, reflects that (3.25) holds for $n=k$. Therefore, (3.25) and (3.24) hold for every $n$. This shows that $\left\{x_{k}\right\}$ is a Cauchy sequence and hence there exists $x^{*} \in \mathbb{B}\left(\bar{x}, \beta_{x^{*}}\right)$ such that $\lim _{k \rightarrow \infty} x_{k}:=x^{*}$. Since $\left(x_{k+1}, w_{k}\right) \in \operatorname{gph} \mathcal{W}_{x_{k}} \cap$ $\left(\mathbb{B}\left(\bar{x}, \frac{r_{\bar{x}}}{2}\right) \times \mathbb{B}(\bar{y}, b)\right)$ and the fact that $\operatorname{gph} \mathcal{W}_{x_{k}} \cap\left(\mathbb{B}\left(\bar{x}, \frac{r_{\bar{x}}}{2}\right) \times \mathbb{B}(\bar{y}, b)\right)$ is closed for each $k=0,1, \ldots$, taking limit $k \rightarrow \infty$ to (3.25). This gives that $0 \in \mathcal{R}_{x^{*}}\left(x^{*}, x^{*}\right)$ and thus $0 \in \mathcal{W}_{x^{*}}\left(x^{*}\right)$, that is, $0 \in f\left(x^{*}\right)+F\left(x^{*}\right)$. This completes the proof.

The following corollary, which is reduced from Theorem 3.1, provides the local convergence of the sequence generated by Algorithm 1.1 in the case when $\bar{x}$ is a solution of (1.1) (i.e. $\bar{y}=0$ ).

Corollary 3.1. Let $\eta>1, u \in X$ and $\bar{x}$ be a solution of (1.1). Let $\mathcal{W}_{\bar{x}}$ be a metrically regular at $(\bar{x}, 0)$ with a positive constant $\kappa$, which have locally closed graph at $(\bar{x}, 0)$. Let $x \in \mathbb{B}\left(\bar{x}, \frac{r_{\bar{x}}}{2}\right)$ 
and $\mathcal{R}_{x}(x, u)$ be partially pseudo-Lipschitz around $((\bar{x}, \bar{x}), 0)$ with a constant $\mu>0$. Suppose that $\mathcal{D} f$ is continuous at $\bar{x}$ with a positive constant $\varepsilon$ such that $\varepsilon(4 \eta+1) \kappa<1-\kappa \mu$ and that

$$
\lim _{x \rightarrow \bar{x}} \mathrm{~d}\left(0, \mathcal{W}_{x}(x)\right)=0 \quad \text { and } \quad \mathrm{d}\left(0, \mathcal{R}_{x}(x, \bar{x})\right) \leq \varepsilon\|x-\bar{x}\| .
$$

Then, for every $k \in \mathbb{N}_{0}, w_{k} \in \mathbb{B}(\bar{y}, b)$ with $w_{k} \in \mathcal{R}_{x}(x, u)$ satisfying

$$
\mathcal{W}_{x}(u) \ni w_{k}
$$

there exists $\hat{\delta}>0$ such that for any sequence $\left\{x_{k}\right\}$ generated by Algorithm 1.1 with initial point $x_{0} \in \mathbb{B}(\hat{\delta}, \bar{x})$ converges linearly to a solution $\bar{x}$ of $(1.1)$, that is, $0 \in f(\bar{x})+F(\bar{x})$.

Proof. By virtue of the metrically regular property of $\mathcal{W}_{\bar{x}}$ at $(\bar{x}, 0)$, we have that there exists constants $\hat{r}_{\bar{x}}>0, r_{0}>0$ and $\kappa>0$ such that

$$
\mathrm{d}\left(x, \mathcal{W}_{\bar{x}}^{-1}(y) \leq \kappa \mathrm{d}\left(y, \mathcal{W}_{\bar{x}}(x)\right) \text { for all } x(x, y) \in\left(\mathbb{B}\left(\bar{x}, \hat{r}_{\bar{x}}\right) \times \mathbb{B}\left(0, r_{0}\right)\right)\right.
$$

and $\operatorname{gph} \mathcal{W}_{\bar{x}} \cap\left(\mathbb{B}\left(\bar{x}, \hat{r}_{\bar{x}}\right) \times \mathbb{B}\left(0, r_{0}\right)\right)$ is closed. Then, for each $0<r_{\bar{x}} \leq \hat{r}_{\bar{x}}$, one has that

$$
\mathrm{d}\left(x, \mathcal{W}_{\bar{x}}^{-1}(y) \leq \kappa \mathrm{d}\left(y, \mathcal{W}_{\bar{x}}(x)\right) \text { for all }(x, y) \in\left(\mathbb{B}\left(\bar{x}, r_{\bar{x}}\right) \times \mathbb{B}\left(0, r_{0}\right)\right),\right.
$$

that is, $\mathcal{W}_{\bar{x}}$ is metrically regular at $(\bar{x}, 0)$ on $\mathbb{B}\left(\bar{x}, r_{\bar{x}}\right) \times \mathbb{B}\left(0, r_{0}\right)$ with constant $\kappa$.

Because $\mathcal{D} f$ is continuous at $\bar{x}$ with constant $\varepsilon$, we can choose $r_{\bar{x}} \in\left(0, \hat{r}_{\bar{x}}\right)$ such that $r_{0}-2 \varepsilon r_{\bar{x}}>0$ and

$$
\left\|\mathcal{D} f(x)-\mathcal{D} f\left(x^{\prime}\right)\right\| \leq \varepsilon \quad \text { for all } \quad x, x^{\prime} \in \mathbb{B}\left(\frac{r_{\bar{x}}}{2}, \bar{x}\right)
$$

Then, define

$$
b:=\min \left\{r_{0}-2 \varepsilon r_{\bar{x}}, \frac{r_{\bar{x}}(1-\kappa \varepsilon)}{4 \kappa}\right\} .
$$

Moreover, the partial pseudo-Lipschitz property of $\mathcal{R}_{x}(x, u)$ at $((\bar{x}, \bar{x}), 0)$ implies that there exist constants $r_{\bar{x}}>0, b>0$ and $\mu>0$ such that $\mathcal{R}_{x}(x, u)$ is partially Lipschitz-like at $((\bar{x}, \bar{x}), 0)$ on $\left(\mathbb{B}\left(\bar{x}, \frac{r_{\bar{x}}}{2}\right) \times \mathbb{B}\left(\bar{x}, \frac{r_{\bar{x}}}{2}\right)\right) \times \mathbb{B}(0, b)$ with constant $\mu$ imply, for all $x, x^{\prime}, x^{\prime \prime} \in \mathbb{B}\left(\bar{x}, \frac{r_{\bar{x}}}{2}\right)$, that

$$
e\left(\mathcal{R}_{x}\left(x, x^{\prime}\right) \cap \mathbb{B}(0, b), \mathcal{R}_{x}\left(x, x^{\prime \prime}\right)\right) \leq \mu\left\|x^{\prime}-x^{\prime \prime}\right\|
$$

Let $\varepsilon \in(0,1)$ be so chosen that $\varepsilon(4 \eta+1) \kappa \leq 1-\kappa \mu$ and

$$
\min \left\{\frac{r_{\bar{x}}}{2}, \frac{b}{3 \varepsilon}, 1, \frac{b \kappa}{1-\kappa \varepsilon}\right\}>0 .
$$

Thus we can choose $\delta \in(0,1]$ such that

$$
\delta \leq \min \left\{\frac{r_{\bar{x}}}{2}, \frac{b}{3 \varepsilon}, \frac{b \kappa}{1-\kappa \varepsilon}\right\} .
$$

Let $x_{0} \in \mathbb{B}(\hat{\delta}, \bar{x})$ and $w_{k} \in \mathbb{B}(\bar{y}, b)$ with $w_{k} \in \mathcal{R}_{x}(x, u)$ such that (3.48) holds. Since (3.47) holds, we can choose $\hat{\delta} \in(0, \delta]$ for which the following inequalities hold:

$$
\mathrm{d}\left(w_{0}, \mathcal{W}_{x_{0}}\left(x_{0}\right)\right)<\varepsilon \delta \quad \text { and } \mathrm{d}\left(0, \mathcal{R}_{x_{0}}\left(x_{0}, \bar{x}\right)\right) \leq \varepsilon\left\|x_{0}-\bar{x}\right\|
$$

Now, one can easily check that all the requirements of Theorem 3.1 are satisfied. Therefore, Theorem 3.1 is applicable to conclude that any sequence $\left\{x_{k}\right\}$ generated by Algorithm 1.1 with starting point $x_{0} \in \mathbb{B}(\hat{\delta}, \bar{x})$ converges to a solution $\bar{x}$ of (1.1). 


\subsection{Quadratic Convergence}

In this subsection, we are devoted to present that if the derivative of $f$ is Lipschitz continuous around $\bar{x}$, then any sequence generated by Algorithm 1.1 converges quadratically.

Theorem 3.2. Suppose that $\mathcal{W}_{\bar{x}}$ is metrically regular at $(\bar{x}, \bar{y})$ on $\mathbb{B}\left(r_{\bar{x}}, \bar{x}\right) \times \mathbb{B}\left(r_{\bar{y}}, \bar{y}\right)$ with constant $\kappa>0$, gph $\mathcal{W}_{\bar{x}} \cap\left(\mathbb{B}\left(r_{\bar{x}}, \bar{x}\right) \times \mathbb{B}\left(r_{\bar{y}}, \bar{y}\right)\right)$ is closed and $\mathcal{D} f$ is Lipschitz continuous on $\mathbb{B}\left(\frac{r_{\bar{x}}}{2}, \bar{x}\right)$ with constant $\ell$. Let $\eta>1$ and $R>0$ be such that

$$
R:=\min \left\{r_{\bar{y}}-2 \ell r_{\bar{x}}^{2}, \frac{r_{\bar{x}}\left(1-\kappa \ell r_{\bar{x}}\right)}{4 \kappa}\right\} .
$$

For every $u \in X$, assume that $\mathcal{R}_{x}(x, u)$ is partially Lipschitz-like at $((\bar{x}, \bar{x}), \bar{y})$ on $\left(\mathbb{B}\left(\bar{x}, \frac{r_{\bar{x}}}{2}\right) \times\right.$ $\left.\mathbb{B}\left(\bar{x}, \frac{r_{\bar{x}}}{2}\right)\right) \times \mathbb{B}(\bar{y}, R)$ with constant $\mu>0$ so that $\kappa\left(\mu+\ell r_{\bar{x}}\right)<1$. Let $\delta>0$ be such that

$$
\begin{aligned}
& \delta \leq \min \left\{\frac{r_{\bar{x}}}{4}, \sqrt{\frac{R}{6 \ell}}, \frac{\kappa R}{1-\kappa \ell r_{\bar{x}}}, 1\right\} ; \\
& (\kappa+1) \ell\left(9 \eta \delta+2 r_{\bar{x}}\right) \leq 2(1-\kappa \mu) ; 2\|\bar{y}\|<\ell \delta^{2} .
\end{aligned}
$$

Suppose that

$$
\lim _{x \rightarrow \bar{x}} \mathrm{~d}\left(\bar{y}, \mathcal{W}_{x}(x)\right)=0 \text { and } \mathrm{d}\left(\bar{y}, \mathcal{R}_{x}(x, \bar{x})\right) \leq \frac{\ell}{2}\|x-\bar{x}\|^{2} .
$$

Then, for every $k \in \mathbb{N}_{0}, t_{k} \in \mathbb{B}(\bar{y}, R)$ with $t_{k} \in \mathcal{R}_{x}(x, u)$ satisfying

$$
\mathcal{W}_{x}(u) \ni t_{k}
$$

there exists some $\hat{\delta}>0$ with an initial point in $\mathbb{B}(\hat{\delta}, \bar{x})$, Algorithm 1.1 generates a sequence, which may not be unique, and any generated sequence $\left\{x_{n}\right\}$ converges quadratically to a solution $x^{*}$ of $(1.1)$.

Proof. According to our assumption, the mapping $\mathcal{W}_{\bar{x}}$ is metrically regular at $(\bar{x}, \bar{y})$ on $\mathbb{B}\left(r_{\bar{x}}, \bar{x}\right) \times \mathbb{B}\left(r_{\bar{y}}, \bar{y}\right)$ with constant $\kappa$ and $\mathcal{D} f$ is Lipschitz continuous on $\mathbb{B}\left(\bar{x}, \frac{r_{\bar{x}}}{2}\right)$ with constant $\ell$. The Lipschitz continuity property of $\mathcal{D} f$ gives, for all $x, x^{\prime} \in \mathbb{B}\left(\bar{x}, \frac{r_{\bar{x}}}{2}\right)$, that

$$
\left\|\mathcal{D} f(x)-\mathcal{D} f\left(x^{\prime}\right)\right\| \leq \ell\left\|x-x^{\prime}\right\| \leq \ell r_{\bar{x}}
$$

Thus, Lemma 3.2 is applicable with $\varepsilon:=\ell r_{\bar{x}}$. Then, applying Lemma 3.2 we obtain, for every $x \in \mathbb{B}\left(\bar{x}, \frac{r_{\bar{x}}}{2}\right)$ and $R$ defined in the statement, that $\mathcal{W}_{x}$ is metrically regular at $(\bar{x}, \bar{y})$ on $\mathbb{B}\left(\bar{x}, \frac{r_{\bar{x}}}{2}\right) \times \mathbb{B}(\bar{y}, R)$ with constant $\gamma:=\frac{\kappa}{1-\kappa \ell r_{\bar{x}}}$, that is,

$$
\mathrm{d}\left(u_{2}, \mathcal{W}_{x}^{-1}\left(v_{2}\right)\right) \leq \gamma \mathrm{d}\left(v_{2}, \mathcal{W}_{x}\left(u_{2}\right)\right) \quad \text { for any } u_{2} \in \mathbb{B}\left(\bar{x}, \frac{r_{\bar{x}}}{2}\right), v_{2} \in \mathbb{B}(\bar{y}, R)
$$

Now, using (3.53) we obtain, for all $u_{1}, v_{1} \in \mathbb{B}\left(\bar{x}, \frac{r_{\bar{x}}}{2}\right)$, that

$$
\begin{aligned}
& \left\|f\left(u_{1}\right)-f\left(v_{1}\right)-\mathcal{D} f\left(v_{1}\right)\left(u_{1}-v_{1}\right)\right\| \\
= & \left\|\int_{0}^{1} \mathcal{D} f\left(v_{1}+\tau\left(u_{1}-v_{1}\right)\right)\left(u_{1}-v_{1}\right) d \tau-\mathcal{D} f\left(v_{1}\right)\left(u_{1}-v_{1}\right)\right\| \\
\leq & \int_{0}^{1}\left\|\mathcal{D} f\left(v_{1}+\tau\left(u_{1}-v_{1}\right)\right)-\mathcal{D} f(v)\right\|\|u-v\| d \tau \\
\leq & \ell\left\|u_{1}-v_{1}\right\|^{2} \int_{0}^{1} \tau d \tau=\frac{\ell}{2}\left\|u_{1}-v_{1}\right\|^{2} .
\end{aligned}
$$


Furthermore, it is given in the statement of the theorem that $\mathcal{R}_{x}(x, u)$ is partial Lipschitzlike at $((\bar{x}, \bar{x}), \bar{y})$ on $\left(\mathbb{B}\left(\bar{x}, \frac{r_{\bar{x}}}{2}\right) \times \mathbb{B}\left(\bar{x}, \frac{r_{\bar{x}}}{2}\right)\right) \times \mathbb{B}(\bar{y}, R)$ with constant $\mu$. This implies, for all $x, x_{1}, x_{2} \in \mathbb{B}\left(\bar{x}, \frac{r_{\bar{x}}}{2}\right)$, that

$$
e\left(\mathcal{R}_{x}\left(x, x_{1}\right) \cap \mathbb{B}(\bar{y}, R), \mathcal{R}_{x}\left(x, x_{2}\right)\right) \leq \mu\left\|x_{1}-x_{2}\right\| .
$$

Moreover, the inequality $\kappa\left(\mu+\ell r_{\bar{x}}\right)<1$, together with $\gamma:=\frac{\kappa}{1-\kappa \ell r_{\bar{x}}}$, gives that $\gamma \mu<1$. Set

$$
\zeta:=\frac{\eta \ell \kappa \delta}{1-\kappa \ell r_{\bar{x}}} \Rightarrow \zeta=\eta \ell \gamma \delta
$$

By (3.50b), one sees that

$$
\kappa \ell\left(9 \eta \delta+2 r_{\bar{x}}\right) \leq(\kappa+1) \ell\left(9 \eta \delta+2 r_{\bar{x}}\right) \leq 2(1-\kappa \mu)<2 .
$$

It follows from (3.57) that

$$
\zeta \leq \frac{2}{9}
$$

On the other hand, with the help of (3.58) and $\eta>1$ we obtain

$$
\frac{\gamma \ell \delta}{1-\gamma \mu}=\frac{\kappa \ell \delta}{1-\kappa\left(\mu+\ell r_{\bar{x}}\right)} \leq \frac{\eta \kappa \ell \delta}{1-\kappa\left(\mu+\ell r_{\bar{x}}\right)}<\frac{2}{9} .
$$

Let $t_{k} \in \mathbb{B}(\bar{y}, R)$ with $t_{k} \in \mathcal{R}_{x}(x, u)$ so that (3.52) holds and $t_{k} \searrow 0$. Select $\hat{\delta} \in(0, \delta]$ and let $x_{0} \in \mathbb{B}(\bar{x}, \hat{\delta})$. Then, for second condition of (3.50b) and first condition of (3.51), we infer that the following conditions hold:

$$
\mathrm{d}\left(t_{0}, \mathcal{W}_{x_{0}}\left(x_{0}\right)\right) \leq \frac{\ell \delta^{2}}{2} \quad \text { and } \mathrm{d}\left(\bar{y}, \mathcal{R}_{x_{0}}\left(x_{0}, \bar{x}\right)\right) \leq \frac{\ell}{2}\left\|x_{0}-\bar{x}\right\|^{2} \quad x \text { for each } x_{0} \in \mathbb{B}(\hat{\delta}, \bar{x}) .
$$

As in the proof for Theorem 3.1, we use induction to show that Algorithm 1.1 generates at least one sequence and any sequence $\left\{x_{k}\right\}$ generated by Algorithm 1.1 satisfies the following assertions:

$$
\left\|s_{k}\right\| \leq \zeta\left(\frac{1}{2}\right)^{2^{k}} \delta
$$

and

$$
\left(x_{k+1}, t_{k}\right) \in \operatorname{gph} \mathcal{W}_{x_{k}} \cap\left(\mathbb{B}\left(\bar{x}, \frac{r_{\bar{x}}}{2}\right) \times \mathbb{B}(\bar{y}, R)\right)
$$

for each $k=0,1, \ldots$. Define

$$
\alpha_{x}:=\frac{\gamma \ell}{1-\gamma \mu}\|x-\bar{x}\|^{2} \quad \text { for each } x \in X .
$$

For each $x \in \mathbb{B}(\bar{x}, 2 \delta)$, it follows from (3.59) that

$$
\alpha_{x}<\frac{4 \gamma \ell \delta^{2}}{1-\gamma \mu}=\frac{4 \gamma \ell \delta}{1-\gamma \mu} \cdot \delta<\delta .
$$

Firstly, we want to show that the point $x_{1}$ exists which satisfy (3.61) and (3.62) for $k=0$. To do this, it is enough to show, by applying Lemma 2.3 to the mappings $\Xi_{x_{0}}$ and $\Upsilon_{x_{0}}$, defined by (3.11) and (3.12) respectively, with $\bar{x}:=\bar{x}$ and $\bar{y}:=\bar{y}$, that

$$
\Lambda_{\triangle}\left(x_{0}\right) \neq \emptyset \text {. }
$$


Now, we check that all assumptions of (2.4)-(2.7) of Lemma 2.3 are hold for the mappings $\Phi:=\Xi_{x_{0}}$ and $\theta:=\Upsilon_{x_{0}}$ with $\kappa:=\gamma, \lambda:=\mu, \bar{x}:=\bar{x}, \bar{y}:=\bar{y}$ and $c:=\alpha_{x_{0}}$.

For $x_{0} \in \mathbb{B}\left(\bar{x}, \alpha_{x_{0}}\right) \subseteq \mathbb{B}(\bar{x}, \delta) \subseteq \mathbb{B}\left(\bar{x}, \frac{r_{\bar{x}}}{2}\right)$, we have from the second condition of (3.60) that

$$
\mathrm{d}\left(\bar{y}, \mathcal{R}_{x_{0}}\left(x_{0}, \bar{x}\right)\right) \leq \frac{\ell}{2}\left\|x_{0}-\bar{x}\right\|^{2} .
$$

This gives, together with (3.11), that

$$
\begin{aligned}
\mathrm{d}\left(\bar{y}, \Xi_{x_{0}}(\bar{x})\right) & =\mathrm{d}\left(\bar{y}, \mathcal{R}_{x_{0}}\left(x_{0}, \bar{x}\right)\right) \leq \frac{\ell}{2}\left\|x_{0}-\bar{x}\right\|^{2} \\
& \leq \frac{\alpha_{x_{0}}(1-\gamma \mu)}{2 \gamma}:=\frac{c(1-\gamma \mu)}{2 \gamma}
\end{aligned}
$$

This yields that the assumption (2.4) of Lemma 2.3 is hold. Furthermore, for any $x \in \mathbb{B}(\bar{x}, 2 \delta)$, we obtain, from (3.50a) and (3.55), that

$$
\begin{aligned}
& \left\|\left(\bar{y}-h_{x_{0}}(x)\right)-\bar{y}\right\| \\
= & \left\|\bar{y}-f(\bar{x})-\mathcal{D} f(\bar{x})(x-\bar{x})+f\left(x_{0}\right)+\mathcal{D} f\left(x_{0}\right)\left(x-x_{0}\right)-\bar{y}\right\| \\
\leq & \left\|f(\bar{x})-f\left(x_{0}\right)-\mathcal{D} f\left(x_{0}\right)\left(\bar{x}-x_{0}\right)\right\|+\left\|\mathcal{D} f\left(x_{0}\right)-\mathcal{D} f(\bar{x})\right\|\|\bar{x}-x\| \\
\leq & \frac{\ell}{2}\left\|\bar{x}-x_{0}\right\|^{2}+\ell\left\|x_{0}-\bar{x}\right\|\|\bar{x}-x\| \leq \frac{5 \ell \delta^{2}}{2} \leq R .
\end{aligned}
$$

Setting $x=\bar{x}$ in (3.66), which gives that

$$
\left\|\left(\bar{y}-h_{x_{0}}(\bar{x})\right)-\bar{y}\right\| \leq \frac{\ell}{2}\left\|\bar{x}-x_{0}\right\|^{2} \leq \frac{\ell \delta^{2}}{2} \leq R .
$$

Therefore, for all $x \in \mathbb{B}(\bar{x}, 2 \delta)$, the above inequalities show that $\bar{y}-h_{x_{0}}(x) \in \mathbb{B}(\bar{y}, R)$. By the construction of $\mathcal{W}_{x}$ in $(3.1)$, we get that

$$
\bar{y}-h_{x_{0}}(\bar{x}) \in \mathcal{W}_{x_{0}}(\bar{x}) \cap \mathbb{B}(\bar{y}, R) .
$$

Thus, by the metric regularity property of $\mathcal{W}_{x_{0}}$ from $(3.17)$, definition of $\Upsilon_{x_{0}}$ in (3.12) and the construction of $h_{x_{0}}$, we obtain, for $x_{0} \in \mathbb{B}\left(\bar{x}, \beta_{x_{0}}\right) \subseteq \mathbb{B}(\bar{x}, \delta) \subseteq \mathbb{B}\left(\bar{x}, \frac{r_{\bar{x}}}{2}\right)$, that

$$
\begin{aligned}
\mathrm{d}\left(\bar{x}, \Upsilon_{x_{0}}(\bar{y})\right) & =\mathrm{d}\left(\bar{x}, \mathcal{W}_{x_{0}}^{-1}(\bar{y})\right) \leq \gamma \mathrm{d}\left(\bar{y}, \mathcal{W}_{x_{0}}(\bar{x})\right) \\
& \leq \gamma\left\|\left(\bar{y}-h_{x_{0}}(\bar{x})\right)-\bar{y}\right\|
\end{aligned}
$$

It follows from (3.67) that

$$
\begin{aligned}
\mathrm{d}\left(\bar{x}, \Upsilon_{x_{0}}(\bar{y})\right) & \leq \gamma\left\|\left(\bar{y}-h_{x_{0}}(\bar{x})\right)-\bar{y}\right\| \leq \frac{\gamma \ell}{2}\left\|\bar{x}-x_{0}\right\|^{2} \\
& =\frac{\alpha_{x_{0}}(1-\gamma \mu)}{2}:=\frac{c(1-\gamma \mu)}{2} .
\end{aligned}
$$

Hence, assertion (2.5) of Lemma 2.3 is hold.

To verify assumptions (2.6) and (2.7) of Lemma 2.3 are hold, let $x^{\prime}, x^{\prime \prime} \in \mathbb{B}\left(\bar{x}, \alpha_{x_{0}}\right)$. Then by the relation $4 \delta \leq r_{\bar{x}}$ in (3.50a) and (3.63), we have $x^{\prime}, x^{\prime \prime} \in \mathbb{B}\left(\bar{x}, \alpha_{x_{0}}\right) \subseteq \mathbb{B}(\bar{x}, \delta) \subseteq \mathbb{B}\left(\bar{x}, \frac{r_{\bar{x}}}{2}\right)$. Moreover, by (3.63) and (3.50a) we have that

$$
\alpha_{x_{0}} \leq \delta \leq \frac{\kappa R}{1-\kappa \ell r_{\bar{x}}} \Rightarrow \frac{\alpha_{x_{0}}}{\gamma} \leq R .
$$


For any $x^{\prime}, x^{\prime \prime} \in \mathbb{B}\left(\bar{x}, \alpha_{x_{0}}\right)$, we have from (3.56) that

$$
\begin{aligned}
& e\left(\Xi_{x_{0}}\left(x^{\prime}\right) \cap \mathbb{B}\left(\bar{y}, \frac{\alpha_{x_{0}}}{\gamma}\right), \Xi_{x_{0}}\left(x^{\prime \prime}\right)\right) \\
\leq & e\left(\Xi_{x_{0}}\left(x^{\prime}\right) \cap \mathbb{B}(\bar{y}, R), \Xi_{x_{0}}\left(x^{\prime \prime}\right)\right) \\
= & e\left(\mathcal{R}_{x_{0}}\left(x_{0}, x^{\prime}\right) \cap \mathbb{B}(\bar{y}, R), \mathcal{R}_{x_{0}}\left(x_{0}, x^{\prime \prime}\right)\right) \\
\leq & \mu\left\|x^{\prime}-x^{\prime \prime}\right\|,
\end{aligned}
$$

which shows that the assertion (2.6) of Lemma 2.3 is satisfied.

Finally, we need to verify that the assertion (2.7) of Lemma 2.3 is also true. To prove this, let $y, y^{\prime} \in \mathbb{B}\left(\bar{y}, \frac{\alpha_{x_{0}}}{\gamma}\right)$. Let $u_{1} \in \mathcal{W}_{x_{0}}^{-1}(y) \cap \mathbb{B}\left(\bar{x}, \alpha_{x_{0}}\right)$. As $\mathcal{W}_{x_{0}}$ is metrically regular at $(\bar{x}, \bar{y})$ on $\mathbb{B}\left(\bar{x}, \frac{r_{\bar{x}}}{2}\right) \times \mathbb{B}(\bar{y}, R)$ with constant $\gamma$, by $(3.54)$ we have that

$$
\mathrm{d}\left(u_{1}, \mathcal{W}_{x_{0}}^{-1}\left(y^{\prime}\right)\right) \leq \gamma \mathrm{d}\left(y^{\prime}, \mathcal{W}_{x_{0}}\left(u_{1}\right)\right) \leq \gamma\left\|y-y^{\prime}\right\|
$$

Taking supremum at $u_{1} \in \mathcal{W}_{x_{0}}^{-1}(y) \cap \mathbb{B}\left(\bar{x}, \frac{r_{\bar{x}}}{2}\right)$ on both sides, we obtain

$$
\sup _{u_{1} \in \mathcal{W}_{x_{0}}^{-1}(y) \cap \mathbb{B}\left(\bar{x}, \frac{r_{\bar{x}}}{2}\right)} \mathrm{d}\left(u_{1}, \mathcal{W}_{x_{0}}^{-1}\left(y^{\prime}\right)\right) \leq \gamma\left\|y-y^{\prime}\right\| .
$$

Combining the definition of excess $e$ and (3.69), it follows that

$$
\begin{aligned}
& e\left(\Upsilon_{x_{0}}(y) \cap \mathbb{B}\left(\bar{x}, \beta_{x_{0}}\right), \Upsilon_{x_{0}}\left(y^{\prime}\right)\right) \\
\leq & e\left(\mathcal{W}_{x_{0}}^{-1}(y) \cap \mathbb{B}\left(\bar{x}, \frac{r_{\bar{x}}}{2}\right), \mathcal{W}_{x_{0}}^{-1}\left(y^{\prime}\right)\right)=\sup _{u_{1} \in \mathcal{W}_{x_{0}}^{-1}(y) \cap \mathbb{B}\left(\bar{x}, \frac{r_{\bar{x}}}{2}\right)} \mathrm{d}\left(u_{1}, \mathcal{W}_{x_{0}}^{-1}\left(y^{\prime}\right)\right) \leq \gamma\left\|y-y^{\prime}\right\| .
\end{aligned}
$$

This shows that assertion (2.7) of Lemma 2.3 is verified. Since all assumptions (2.4)-(2.7) of Lemma 2.3 are satisfied, Lemma 2.3 is applicable to conclude the existence of fixed points $\hat{x} \in \mathbb{B}\left(\bar{x}, \alpha_{x_{0}}\right)$ and $\hat{y} \in \mathbb{B}\left(\bar{y}, \frac{\alpha_{x_{0}}}{\gamma}\right)$ such that

$$
\hat{y} \in \Xi_{x_{0}}(\hat{x}) \text { and } \hat{x} \in \Upsilon_{x_{0}}(\hat{y}) \text {. }
$$

The second inclusion in (3.70) translates to $\hat{y} \in \mathcal{W}_{x_{0}}(\hat{x})$, that is, $\hat{y} \in f\left(x_{0}\right)+\mathcal{D} f\left(x_{0}\right)\left(\hat{x}-x_{0}\right)+$ $F(\hat{x})$. This, together with the inclusion $\hat{y} \in \Xi_{x_{0}}(\hat{x})=\mathcal{R}_{x_{0}}\left(x_{0}, \hat{x}\right)$ by (3.11) and (3.70), implies that

$$
\hat{y} \in f\left(x_{0}\right)+\mathcal{D} f\left(x_{0}\right)\left(\hat{x}-x_{0}\right)+F(\hat{x}) \cap \mathcal{R}_{x_{0}}\left(x_{0}, \hat{x}\right)
$$

and therefore, (3.64) is hold. Thus, we can choose $s_{0}$ such that $s_{0} \in \Lambda_{\triangle}\left(x_{0}\right)$ and

$$
\left\|s_{0}\right\| \leq \eta \mathrm{d}\left(0, \Lambda_{\triangle}\left(x_{0}\right)\right)
$$

Thus, Algorithm 1.1 ensure that $x_{1}:=x_{0}+s_{0}$ is defined. For such $s_{0}$, we obtain from (3.2) that

$$
\Lambda_{\triangle}\left(x_{0}\right):=\left\{s_{0} \in X: \mathcal{W}_{x_{0}}\left(x_{0}+s_{0}\right) \cap \mathcal{R}_{x_{0}}\left(x_{0}, x_{0}+s_{0}\right) \neq \emptyset\right\} .
$$

Since (3.52) holds for every $t_{k} \in \mathbb{B}(\bar{y}, R)$ with $t_{k} \in \mathcal{R}_{x}(x, u)$, we have that $t_{0} \in \mathcal{W}_{x_{0}}\left(x_{0}+s_{0}\right) \Rightarrow$ $x_{0}+s_{0} \in \mathcal{W}_{x_{0}}^{-1}\left(t_{0}\right)$. This gives from (3.72) that

$$
\Lambda_{\triangle}\left(x_{0}\right)=\left\{s_{0} \in X: x_{0}+s_{0} \in \mathcal{W}_{x_{0}}^{-1}\left(t_{0}\right)\right\} .
$$


Combining (3.72) and (3.73), we obtain that

$$
\mathrm{d}\left(0, \Lambda_{\triangle}\left(x_{0}\right)\right)=\mathrm{d}\left(x_{0}, \mathcal{W}_{x_{0}}^{-1}\left(t_{0}\right)\right)
$$

Linking the metrically regular property of $\mathcal{W}_{x_{0}}$ from (3.54) with constant $\gamma$, we get, from (3.71) and (3.74), that

$$
\left\|s_{0}\right\| \leq \eta \mathrm{d}\left(x_{0}, \mathcal{W}_{x_{0}}^{-1}\left(t_{0}\right)\right) \leq \eta \gamma \mathrm{d}\left(t_{0}, \mathcal{W}_{x_{0}}\left(x_{0}\right)\right) .
$$

This gives, for first inequality in (3.60), that

$$
\left\|s_{0}\right\| \leq \eta \gamma \mathrm{d}\left(t_{0}, \mathcal{W}_{x_{0}}\left(x_{0}\right)\right) \leq \frac{\eta \gamma \ell}{2} \delta^{2}=\frac{\zeta \delta}{2},
$$

which implies that (3.61) holds for $k=0$.

Since $\zeta \leq \frac{2}{9},(3.75)$ gives that

$$
\left\|x_{1}-x_{0}\right\|=\left\|s_{0}\right\| \leq \frac{\zeta \delta}{2}<\delta .
$$

Consequently, we obtain, for $x_{0} \in \mathbb{B}(\bar{x}, \delta)$, that

$$
\left\|x_{1}-\bar{x}\right\| \leq\left\|x_{1}-x_{0}\right\|+\left\|x_{0}-\bar{x}\right\|<2 \delta .
$$

This yields that $x_{1} \in \mathbb{B}(\bar{x}, 2 \delta) \subseteq \mathbb{B}\left(\bar{x}, \frac{r_{\bar{x}}}{2}\right)$. Because of $x_{1}:=x_{0}+s_{0}$ is defined, from (3.73) we get that

$$
\Lambda_{\triangle}\left(x_{0}\right)=\left\{s_{0} \in X: x_{1} \in \mathcal{W}_{x_{0}}^{-1}\left(t_{0}\right)\right\} .
$$

This shows that (3.62) holds for $k=0$ with $x_{1} \in \mathbb{B}\left(\bar{x}, \frac{r_{\bar{x}}}{2}\right)$ and $t_{0} \in \mathbb{B}(\bar{y}, R)$.

We proceed by induction. Actually, we are going to show that Algorithm 1.1 generates at least one sequence, which satisfies (3.61) and (3.62) for all $n$. To finish this, we assume that the points $x_{0}, x_{1}, \ldots, x_{k+1}$ are constructed by Algorithm 1.1 with initial point $x_{0}$ such that (3.61) and (3.62) hold for $n=0,1, \ldots, k-1$ and verify that (3.61) and (3.62) are also hold for $k=n$. Since (3.61) and (3.62) are true for $k=0,1, \ldots, n-1$, by Algorithm 1.1, $x_{n}:=x_{n-1}+s_{n-1}$ is defined and thus $x_{n} \in \mathbb{B}(\bar{x}, 2 \delta) \subseteq \mathbb{B}\left(\bar{x}, \frac{r_{\bar{x}}}{2}\right)$. Now, if we use almost same arguments that we did for the case when $k=0$, one can easily apply Lemma 2.3 to the mappings $\Phi:=\Xi_{x_{n}}$ and $\theta:=\Upsilon_{x_{n}}$ with $\kappa:=\gamma, \lambda:=\mu, \bar{x}:=\bar{x}, \bar{y}:=\bar{y}$ and $c:=\alpha_{x_{n}}$ and show that $\Lambda_{\triangle}\left(x_{n}\right) \neq \emptyset$. Then, choose $s_{n}$ such that $s_{n} \in \Lambda_{\triangle}\left(x_{n}\right)$ and

$$
\left\|s_{n}\right\| \leq \eta \mathrm{d}\left(0, \Lambda_{\triangle}\left(x_{n}\right)\right)
$$

By Algorithm 1.1, $x_{n+1}:=x_{n}+s_{n}$ is defined. By the choice of $s_{n}$, we have from (3.2) that

$$
\Lambda_{\triangle}\left(x_{n}\right):=\left\{s_{n} \in X: \mathcal{W}_{x_{n}}\left(x_{n}+s_{n}\right) \cap \mathcal{R}_{x_{n}}\left(x_{n}, x_{n}+s_{n}\right) \neq \emptyset\right\} .
$$

It is given that, for every $t_{k} \in \mathbb{B}(\bar{y}, R)$ with $t_{k} \in \mathcal{R}_{x}(x, u)$, (3.52) holds. Therefore, we conclude that $t_{n} \in \mathcal{W}_{x_{n}}\left(x_{n}+s_{n}\right) \Rightarrow x_{n}+s_{n} \in \mathcal{W}_{x_{n}}^{-1}\left(t_{n}\right)$. It follows from (3.77) that

$$
\Lambda_{\triangle}\left(x_{n}\right)=\left\{s_{n} \in X: x_{n}+s_{n} \in \mathcal{W}_{x_{n}}^{-1}\left(t_{n}\right)\right\} .
$$

Thus, (3.78) gives that

$$
\mathrm{d}\left(0, \Lambda_{\triangle}\left(x_{n}\right)\right)=\mathrm{d}\left(x_{n}, \mathcal{W}_{x_{n}}^{-1}\left(t_{n}\right)\right)
$$


On the other hand, since $x_{n-1}, x_{n} \in \mathbb{B}(\bar{x}, 2 \delta) \subseteq \mathbb{B}\left(\bar{x}, \frac{r_{\bar{x}}}{2}\right)$ and $t_{n} \in \mathbb{B}(\bar{y}, R)$, we get, from (3.6) and (3.7), that

$$
\left.t_{n}-Z\left(x_{n-1}, x_{n}\right) \in \mathcal{W}_{x_{n}}\left(x_{n}\right)\right) .
$$

Now, we will use the metrically regular property of $\mathcal{W}_{x_{n}}$ from $(3.54)$ with constant $\gamma$. Thus, from (3.76) we obtain that

$$
\begin{aligned}
\left\|s_{n}\right\| & \leq \eta \mathrm{d}\left(x_{n}, \mathcal{W}_{x_{n}}^{-1}\left(t_{n}\right)\right) \leq \eta \gamma \mathrm{d}\left(t_{n}, \mathcal{W}_{x_{n}}\left(x_{n}\right)\right) \\
& \leq \eta \gamma\left\|t_{n}-\left(t_{n}-Z\left(x_{n-1}, x_{n}\right)\right)\right\| \\
& \leq \eta \gamma\left\|f\left(x_{n}\right)-f\left(x_{n-1}\right)-\mathcal{D} f\left(x_{n-1}\right)\left(x_{n}-x_{n-1}\right)\right\| \\
& \leq \frac{\eta \gamma \ell}{2}\left\|x_{n}-x_{n-1}\right\|^{2} \leq \frac{\eta \gamma \ell}{2}\left(\zeta\left(\frac{1}{2}\right)^{2^{n-1}} \delta\right)^{2} \\
& =\frac{\eta \gamma \ell \delta}{2}\left(\zeta^{2}\left(\frac{1}{2}\right)^{2^{n}}\right) \delta \leq \zeta\left(\frac{1}{2}\right)^{2^{n}} \delta .
\end{aligned}
$$

This implies that (3.61) holds for each $k=n$. Moreover, since $\zeta \leq \frac{2}{9}$, using (3.79) we obtain that

$$
\left\|x_{n+1}-\bar{x}\right\| \leq \sum_{i=0}^{n}\left\|s_{i}\right\|+\left\|x_{0}-\bar{x}\right\| \leq \delta \sum_{i=0}^{n}\left(\frac{1}{2}\right)^{2^{i}}+\delta \leq 2 \delta,
$$

which shows that $x_{n+1} \in \mathbb{B}(\bar{x}, 2 \delta) \subseteq \mathbb{B}\left(\bar{x}, \frac{r_{\bar{x}}}{2}\right)$. Furthermore, we obtain from (3.77) that

$$
\Lambda_{\triangle}\left(x_{n}\right)=\left\{s_{n} \in X: x_{n+1} \in \mathcal{W}_{x_{n}}^{-1}\left(t_{n}\right)\right\} \text {. }
$$

This, together with $x_{n+1} \in \mathbb{B}\left(\bar{x}, \frac{r_{\bar{x}}}{2}\right)$ and $t_{n} \in \mathbb{B}(\bar{y}, R)$, gives that (3.62) holds for $k=n$. Therefore, (3.61) and (3.62) hold for every $k$. Therefore, $\left\{x_{k}\right\}$ is a Cauchy sequence and hence there exists $x^{*} \in \mathbb{B}\left(\bar{x}, \alpha_{x_{k}}\right)$ such that $\lim _{k \rightarrow \infty} x_{k}:=x^{*}$. Since $\left(x_{k+1}, t_{k}\right) \in \operatorname{gph} \mathcal{W}_{x_{k}} \cap\left(\mathbb{B}\left(\bar{x}, \frac{r_{\bar{x}}}{2}\right) \times\right.$ $\mathbb{B}(\bar{y}, R))$ and the fact that $\operatorname{gph} \mathcal{W}_{x_{k}} \cap\left(\mathbb{B}\left(\bar{x}, \frac{r_{\bar{x}}}{2}\right) \times \mathbb{B}(\bar{y}, R)\right)$ is closed for each $k=0,1, \ldots$, taking limit $k \rightarrow \infty$ to (3.62) which gives that $0 \in \mathcal{R}_{x^{*}}\left(x^{*}, x^{*}\right)$ and thus $0 \in \mathcal{W}_{x^{*}}\left(x^{*}\right)$, that is, $0 \in f\left(x^{*}\right)+F\left(x^{*}\right)$. This completes the proof.

If $\bar{x}$ is a solution of (1.1) (i.e. $\bar{y}=0$ ), Theorem 3.2 is reduced to the following corollary which provides the local quadratic convergence of the sequence generated by restricted inexact Newton-type method. The proof of this corollary is similar to the proof of Corollary 3.1 and so we omitted its proof here.

Corollary 3.2. Suppose that $\eta>1$ and $\bar{x}$ is a solution of (1.1). Assume that $\mathcal{W}_{\bar{x}}$ is metrically regular at $(\bar{x}, 0)$ with constant $\kappa>0$ and $g \mathrm{ph} \mathcal{W}_{\bar{x}}$ is locally closed. Let $\mathcal{D} f$ be Lipschitz continuous around $\bar{x}$ with constant $\ell$. Let $x \in \mathbb{B}\left(\bar{x}, \frac{r_{\bar{x}}}{2}\right)$ and assume that, for every $u \in X, \mathcal{R}_{x}(x, u)$ is partially Lipschitz-like at $((\bar{x}, \bar{x}), 0)$ with constant $\mu>0$ so that $\kappa\left(\mu+\ell r_{\bar{x}}\right)<1$. Suppose that

$$
\lim _{x \rightarrow \bar{x}} \mathrm{~d}\left(0, \mathcal{W}_{x}(x)\right)=0 \text { and } \mathrm{d}\left(0, \mathcal{R}_{x}(x, \bar{x})\right) \leq \frac{\ell}{2}\|x-\bar{x}\|^{2} .
$$

Then, for every $k \in \mathbb{N}_{0}, t_{k} \in \mathbb{B}(\bar{y}, R)$ with $t_{k} \in \mathcal{R}_{x}(x, u)$ satisfying

$$
\mathcal{W}_{x}(u) \ni t_{k}
$$

there exists some $\hat{\delta}>0$ such that any sequence $\left\{x_{n}\right\}$ generated by Algorithm 1.1 with an initial point in $\mathbb{B}(\hat{\delta}, \bar{x})$ converges quadratically to a solution $\bar{x}$ of $(1.1)$. 


\section{Application and Numerical Experiment}

In this section, we will provide an application of restricted inexact Newton-type method to variational inequality and a numerical example which illustrates the theoretical result.

\subsection{Application to Variational Inequality}

Let $f: \mathbb{R}^{n} \rightarrow \mathbb{R}^{n}$ be a continuously differentiable function and $C$ be a nonempty, closed and convex subset of $\mathbb{R}^{n}$. We consider the following variational inequality problem of finding a point $x \in C$ satisfying

$$
\langle f(x), u-x\rangle \leq 0 \quad \text { for all } u \in C .
$$

Let $N_{C}: \mathbb{R}^{n} \rightrightarrows \mathbb{R}^{n}$ be a normal cone mapping to the convex set $C$ defined by

$$
N_{C}(x)= \begin{cases}\{v:\langle v, u-x\rangle \leq 0, & \forall u \in C\} \text { for } x \in C \\ \emptyset, & \text { otherwise }\end{cases}
$$

We then observe that the classical variational inequality problem (4.1) for $f$ and $C$ is equivalent to the generalized equation to having $x \in X$ such that

$$
f(x)+N_{C}(x) \ni 0 .
$$

Let $P_{C}: \mathbb{R}^{n} \rightrightarrows C$ be a projection mapping which is a continuous function. It is very well known that the following simple connection between the normal cone mapping $N_{C}$ and the projection mapping $P_{C}$ :

$$
v \in N_{C}(x) \Longleftrightarrow P_{C}(x+v)=x .
$$

Let $M(x)=P_{C}(x-f(x))-x=0$. Then the variational inequality (4.2) can actually be written as an equation of the following form:

$$
f(x)+N_{C}(x) \ni 0 \Longleftrightarrow M(x)=0 .
$$

Denote $\mathcal{W}_{x}^{\prime}(\cdot)=f(x)+\mathcal{D} f(x)(\cdot-x)+N_{C}(\cdot)$. Let $\eta_{k}$ be scalars and set $\mathcal{R}_{x}(x, \cdot)=\mathbb{B}\left(0, \eta_{k}\|M(x)\|\right)$, where $\eta_{k} \searrow 0$ and $\|M(x)\|$ is the residual $\mathrm{d}\left(0, \mathcal{W}_{x}^{\prime}(\cdot)\right)$. Therefore, we obtain, for $u \in \mathbb{R}^{n}$, that

$$
\begin{aligned}
& \mathrm{d}\left(0, \mathcal{W}_{x}^{\prime}(u)\right) \leq \eta_{k}\|M(x)\| \\
& \quad \Longrightarrow\left(f(x)+\mathcal{D} f(x)(u-x)+N_{C}(u)\right) \cap \mathbb{B}\left(0, \eta_{k}\|M(x)\|\right) \neq \emptyset .
\end{aligned}
$$

Assume that

$$
\lim _{x \rightarrow \bar{x}} \mathrm{~d}\left(\bar{y}, \mathcal{W}_{x}^{\prime}(x)\right)=0 .
$$

Suppose that $(3.16)$ holds with $\mathcal{W}_{x}^{\prime}(\cdot)$. By the continuity property of $\mathcal{D} f$ with constant $\varepsilon$, we obtain

$$
\begin{aligned}
& \mathrm{d}\left(\bar{y}, \mathbb{B}\left(0, \eta_{k}\|M(x)\|\right)\right) \\
= & \min _{y \in \mathbb{B}\left(0, \eta_{k}\|M(x)\|\right)}\|\bar{y}-y\|=\min _{y \in \mathcal{W}_{x}^{\prime}(\bar{x})}\|\bar{y}-y\|=\mathrm{d}\left(\bar{y}, \mathcal{W}_{x}^{\prime}(\bar{x})\right) \\
\leq & \|f(\bar{x})-f(x)-\mathcal{D} f(x)(\bar{x}-x)\| \leq \varepsilon\|x-\bar{x}\| .
\end{aligned}
$$

For variational inequality problem (4.2), we can thus rewrite (1.6) as follows:

$$
S(x):=\left\{d \in \mathbb{R}^{n}:\left(f(x)+\mathcal{D} f(x) d+N_{C}(x+d)\right) \cap \mathbb{B}\left(0, \eta_{x}\|M(x)\|\right) \neq \emptyset\right\} .
$$

Then we obtain the following Algorithm 4.1, which is the restricted inexact Newton-type method for solving (4.2): 
Algorithm 4.1. (RINM for Variational Inequality)

Step 1. Let $\eta \in[1, \infty)$ and given $x_{0} \in X$, and put $k:=0$.

Step 2. If $0 \in S\left(x_{)}\right.$, that is, $\left(f\left(x_{k}\right)+N_{C}\left(x_{k}\right)\right) \cap \mathbb{B}\left(0, \eta_{x_{k}}\left\|M\left(x_{k}\right)\right\|\right) \neq \emptyset$, then stop; otherwise go to Step 3.

Step 3. If $0 \notin S\left(x_{k}\right)$, choose $d_{k}$ such that $d_{k} \in S\left(x_{k}\right)$ and

$$
\left\|d_{k}\right\| \leq \eta \mathrm{d}\left(0, S\left(x_{k}\right)\right) .
$$

Step 4. Update by $x_{k+1}:=x_{k}+d_{k}$.

Step 5. Update by $k:=k+1$ and go to Step 2 .

Let $f$ in (4.2) be Lipschitz continuous in $\mathbb{B}\left(\bar{x}, \frac{r_{\bar{x}}}{2}\right)$ with Lipschitz constant $\ell$. Then by the nonexpensiveness of the projection mapping $P_{C}$ on $C$, we have that $M$ is Lipschitz continuous with Lipschitz constant $\mu>\ell+2$. It is obvious that (4.4) together with (4.5) imply the condition (3.15). Now, it is our routine to check that all the cases described in Theorem 3.1 are fulfiled and hence we obtain from Theorem 3.1 that Algorithm 4.1 guarantees to generate at least one sequence with starting point close to the initial point which is linearly convergent to the the solution of (4.2).

\subsection{Numerical Experiment}

In order to illustrate the theoretical result of RINM, we consider the following example.

Example 4.1. Let $X=Y=\mathbb{R}, x_{0}=1.1, \eta=3, \kappa=0.318, \varepsilon=0.67, w=0.00001$, and $\lambda=$ 0.404 . Let $f: \mathbb{R} \rightarrow \mathbb{R}, F: \mathbb{R} \rightrightarrows \mathbb{R}$ and a sequence of set-valued mappings $\mathcal{R}_{x}: \mathbb{R} \times \mathbb{R} \rightrightarrows \mathbb{R}$ be defined, respectively, by, for all $x, u \in \mathbb{R}$,

$$
\begin{aligned}
& f(x)=x^{2}-x-4 ; \quad F(x)=\{-2 x,-3\} \quad \text { and } \\
& \mathcal{R}_{x}(x, u)=\left\{x^{2}-x-2(2+u)+(2 x-1)(u-x), x^{2}-x-7+(2 x-1)(u-x)\right\} .
\end{aligned}
$$

Then Algorithm 1.1 generates a sequence which converges linearly to $x^{*}=-1$ and $x^{*}=3.1926$ with initial point at $x_{0}=1.1$.

Solution: Note that

$$
(f+F)(x)=\left\{x^{2}-3 x-4, x^{2}-x-7\right\} \quad \text { for } x \in \mathbb{R} .
$$

Then, it is evident that

$$
(f+F)^{-1}(y)=\{(3 \pm \sqrt{4 y+25}) / 2,(1 \pm \sqrt{4 y+29}) / 2\} .
$$

Taking positive sign and then we obtain that

$$
\mathrm{d}\left(x,(f+F)^{-1}(y)\right)=\inf \{|x-(3+\sqrt{4 y+25}) / 2|,|x-(1+\sqrt{4 y+29}) / 2|\}
$$

and

$$
\mathrm{d}(y,(f+F)(x))=\inf \left\{\left|x^{2}-3 x-4-y\right|,\left|x^{2}-x-7-y\right|\right\} .
$$


Table 4.1: Numerical Results for Example 1.

\begin{tabular}{|c|c|c|c|c|}
\hline \multirow{2}{*}{ Iterations } & \multicolumn{2}{|c|}{$(f+F)(x)=x^{2}-3 x-4$} & \multicolumn{2}{c|}{$(f+F)(x)=x^{2}-x-7$} \\
\cline { 2 - 5 } & $x_{k}$ & $(f+F)\left(x_{k}\right)$ & $x_{k}$ & $(f+F)\left(x_{k}\right)$ \\
\hline 1 & 1.1000 & -6.0900 & 1.1000 & -6.8900 \\
\hline 2 & -6.5125 & 57.9504 & 6.8417 & 32.9668 \\
\hline 3 & -2.8963 & 13.0772 & 4.2425 & 6.7560 \\
\hline 4 & -1.4090 & 2.2121 & 3.3398 & 0.8147 \\
\hline 5 & -1.0287 & 0.1446 & 3.1964 & 0.0206 \\
\hline 6 & -1.0002 & 0.0008 & 3.1926 & 0.0000 \\
\hline 7 & -1.0000 & 0.0000 & - & - \\
\hline
\end{tabular}

Take $x=c$ and $y=1+c$. Then

$$
\begin{aligned}
& \frac{\mathrm{d}\left(x,(f+F)^{-1}(y)\right)}{\mathrm{d}(y,(f+F)(x))} \\
= & \frac{\inf \left\{\left|\frac{2 c-(3+\sqrt{4 c+29})}{2}\right|,\left|\frac{2 c-(1+\sqrt{4 c+33})}{2}\right|\right\}}{\inf \left\{\left|c^{2}-4 c-5\right|,\left|c^{2}-2 c-8\right|\right\}} \\
\leq & \frac{2.251}{8}=0.318, \quad \text { as } c \rightarrow 1 .
\end{aligned}
$$

This implies that $f+F$ is metrically regular around $(1,2)$ with constant $\kappa=0.318$. First, we consider the set-valued mapping $(f+F)(x)=\left\{x^{2}-3 x-4\right\}$ and corresponding this, we have that $\mathcal{R}_{x}(x, u)=\left\{x^{2}-x-2(2+u)+(2 x-1)(u-x)\right\}$. Observe that $f+F$ has a closed graph at $(\bar{x}, \bar{y})$ with $\bar{x}=1$ and $\bar{y}=2$. Thus, $(1,-6) \in \operatorname{gph}(f+F)$. Suppose that $\mathcal{D} f$ is continuous around $\bar{x}=1$ with constant $\varepsilon=0.67$. Then, by Lemma $3.2, \mathcal{W}_{x}$ is metrically regular around $(1,2)$ with constant $\lambda=\frac{\kappa}{1-\kappa \varepsilon}=0.404$. Also, suppose that $\mathcal{R}_{x}(x, \cdot)$ is partially Lipschitz-like at $((1,2), 2)$ with constant $\mu=0.42$ so that $\kappa(\mu+\varepsilon)<1$. Therefore, the assumptions of Theorem 3.1 are hold. Since $w \in \mathcal{R}_{x}(x, u)$ satisfies $w \in \mathcal{W}_{x}(u)$, from (3.44) we have that

$$
\Lambda_{\triangle}\left(x_{k}\right)=\left\{s_{k} \in \mathbb{R}: s_{k}=\frac{w-x_{k}^{2}+3 x_{k}+4}{2 x_{k}-3}\right\} .
$$

On the other hand, if $\Lambda_{\triangle}\left(x_{k}\right) \neq \emptyset$ and $s_{k}=x_{k+1}-x_{k}$, we obtain that

$$
x_{k+1}=\frac{x_{k}^{2}+w+4}{2 x_{k}-3} \text {. }
$$

Moreover, from (3.45), we have that $\left\|s_{k}\right\| \leq \eta \lambda \varepsilon\left\|s_{k-1}\right\|$.

Hence, for the given values of $\eta, \varepsilon, \lambda, w$ and $\kappa$, one sees that Algorithm 1.1 generates a linearly convergent sequence with initial point $x_{0}=1.1$ in a neighborhood of $\bar{x}=1$ which converges to $x^{*}=-1$. Now if we consider the set-valued mapping $(f+F)(x)=\left\{x^{2}-x-7\right\}$ and its corresponding inexactness set-valued mapping $\mathcal{R}_{x}(x, u)=\left\{x^{2}-x-7+(2 x-1)(u-x)\right\}$ and apply the same procedure that we did for above, we can show that Algorithm 1.1 generates a linearly convergent sequence with initial point $x_{0}=1.1$ in a neighborhood of $\bar{x}=1$ which converges to $x^{*}=3.1926$. The numerical results are shown in Table 4.1 which are obtained by using Mat lab program and this indicates that the generalized equation $f(x)+F(x) \ni 0$ has two solutions: $x^{*}=-1$ and $x^{*}=3.1926$. The graphs of $f+F$ are plotted in Fig. 4.1. 

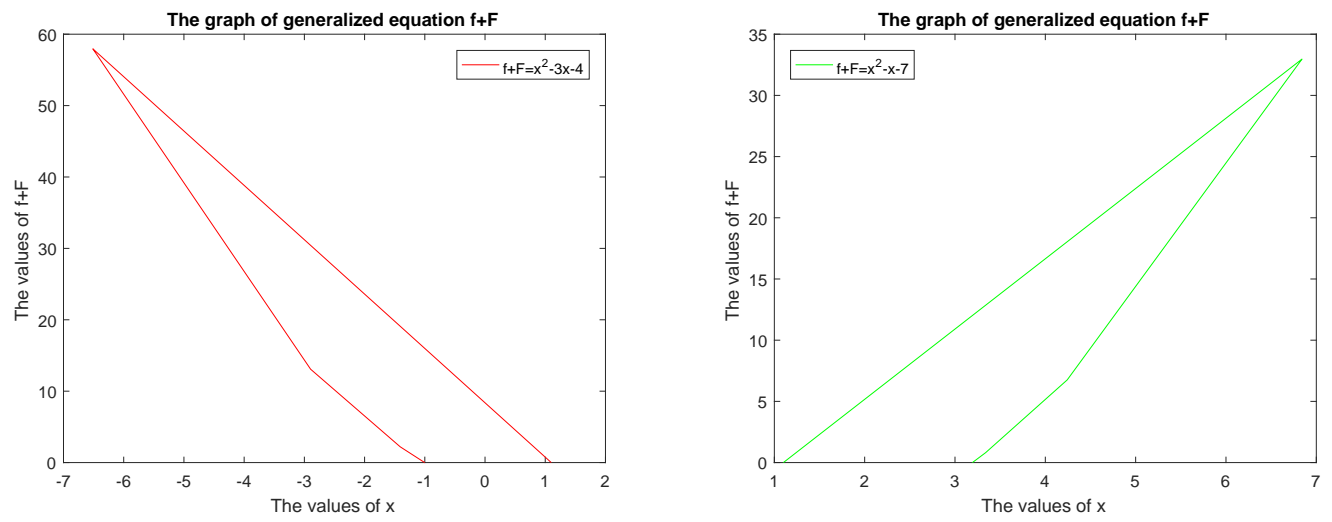

Fig. 4.1. Linear rate of convergence of Algorithm 1 at -1 and 3.1926.

\section{Concluding Remarks}

For solving generalized equation (1.1), semilocal and local convergence results for restricted inexact Newton-type method are presented when $\eta>1$. Under the assumptions that when $\mathcal{W}_{\bar{x}}$ is metrically regular and $\mathcal{D} f$ is continuous, we have shown that the sequence generated by Algorithm 1.1 converges linearly. On the other hand, if $\mathcal{D} f$ is Lipschitz continuous, we have presented the quadratic convergence of the sequence generated by restricted inexact Newton-type method. An application to variational inequality for generalized equation (1.1) is presented. Finally, we have provided a numerical experiment that illustrates the theoretical results. In the framework of generalized equation (1.1), it seems that the restricted inexact Newton-type method and the established convergence results are new contributions and consequently, this study improves and extends the result corresponding to [25].

Acknowledgments. This research work is supported by CAS-President International Fellowship Initiative (PIFI) from the Academy of Mathematics and Systems Science, Chinese Academy of Sciences, Beijing, China.

\section{References}

[1] S. Adly, R. Cibulka and H.V. Ngai, Newton's method for solving inclusions using set-valued approximations, SIAM J. Optim., 25:1 (2015), 159-184.

[2] S. Adly, H.V. Ngai and V.V. Nguyen, Newton's method for solving generalized equations: Kantorovich's and Smale's approaches, J. Math. Anal. Appl., 439:1 (2016), 396-418.

[3] F.J. Aragon Artacho, M. Belyakov, A.L. Dontchev and M. Lopez, Local convergence of quasiNewton methods under metric regularity, Comput. Optim. Appl., 58 (2014), 225-247.

[4] F.J. Aragón Artacho, A.L. Dontchev, M. Gaydu, M.H. Geoffroy and V.M. Veliov, Metric regularity of Newton's iteration, SIAM J. Control Optim., 49:2 (2011), 339-362.

[5] I.K. Argyros, Advances in the Efficiency of Computational Methods and Applications, River Edge, NJ World Scientific, 2000.

[6] I.K. Argyros, On a nonsmooth version of Newton's method based on Hölderian assumptions, International Journal of Computer Mathematics, 84:12 (2007), 1747-1756.

[7] I.K. Argyros and S. Hilout, Inexact Newton-type methods, J. Complexity, 26 (2010), 577-590.

[8] I.K. Argyros and L.U. Uko, A semilocal convergence analysis of an inexact Newton method using recurrent relations, Punjab University Journal of Mathematics, 45 (2013), 25-32. 
[9] I.K. Argyros and S. Hilout, Local convergence of Newton-like methods for generalized equations, Appl. Math. and Comp., 197 (2008), 507-514.

[10] J.P. Aubin, Lipschitz behavior of solutions to convex minimization problems, Math. Oper. Res., 9 (1984), 87-111.

[11] J.P. Aubin and H. Frankowska, Set-valued analysis, Birkhäuser, Boston, 1990.

[12] S. Burnet, C. Jean-Alexis and A. Piétrus, Exact and inexact Hummel-Seebeck type method for variational inclusions, Advances in Analysis, 2:4 (2017), 257-266.

[13] R. Cibulka, A.L. Dontchev and M. H. Geoffroy, Inexact Newton methods and Dennis-Moré theorems for nonsmooth generalized equations, SIAM J. Control Optim., 53:2 (2015), 1003-1019.

[14] R. Cibulka, A.L. Dontchev, J. Preininger, T. Roubal and V. M. Veliov, Kantorovich-type theorems for generalized equations, J. Convex Anal., 25:2 (2018), 459-486.

[15] R.S. Dembo, S.C. Eisenstat and T. Steihaug, Inexact Newton Methods, SIAM J. Numer. Anal., 19 (1982), 400-408.

[16] A.L. Dontchev, Local convergence of the Newton method for generalized equation, C.R.A.S Paris Series I, 322 (1996), 327-331.

[17] A.L. Dontchev, The Graves theorem revisited, J. Convex Anal., 3 (1996), 45-53.

[18] A.L. Dontchev, Uniform convergence of the Newton method for Aubin continuous maps, Serdica Math. J., 22 (1996), 385-398.

[19] A.L. Dontchev, Local analysis of a Newton-type method based on partial linearization, Lectures in Applied Mathematics, 32 (1996), 295-306.

[20] A.L. Dontchev and H. Frankowska, Lyusternik-Graves theorem and fixed points II, J. Convex Anal., 19 (2012), 955-973.

[21] A.L. Dontchev, A.S. Lewis and R.T. Rockafellar, The radius of metric regularity, Trans AMS, 355 (2002), 493-517.

[22] A.L. Dontchev, M. Quincampoix and Zlateva, Aubin criterion for metric regularity, J. Convex Anal., 13:2 (2006), 281-297.

[23] A.L. Dontchev and R.T. Rockafellar, Regularity and conditioning of solution mappings in variational analysis, Set-valued Anal., 12 (2004), 79-109.

[24] A.L. Dontchev and R.T. Rockafellar, Implicit functinos and solution mappings: A view from variational analysis, Springer, Second edition, 2014.

[25] A.L. Dontchev and R.T. Rockafellar, Convergence of inexact Newton methods for generalized equations, Math. Program. Ser. B, 139 (2013), 115-137.

[26] A.L. Dontchev and V.M. Veliov, Metric regularity under approximations, Control Cyber-net., 38 (2009), 1283-1303.

[27] O.P. Ferreira and G.N. Silva, Inexact Newton's method to nonlinear functions with values in a cone, arXiv, in press. arXiv:1510.01947, 2015.

[28] M.C. Ferris and J.S. Pang, Engineering and economic applications of complementarity problems, SIAM Rev., 39 (1997), 669-713.

[29] A.D. Ioffe Metric regularity and subdifferential calculus, Russian Math Surveys, 55 (2000), 501558.

[30] A.F. Izmailov, M.V. Solodov, Inexact Josephy-Newton framework for generalized equations and its applications to local analysis of Newtonian methods for constrained optimization, Comput. Optim. Appl., 46 (2010), 347-368.

[31] A.F. Izmailov, and M.V. Solodov, Newton-type methods for optimization and variational problems, Springer Series in Operations Research and Financial Engineering, Springer, Cham , 2014.

[32] D. Klatte and B. Kummer, Approximations and generalized Newton methods, Mathematical Programming, 168:1-2 (2018), 673-716.

[33] D. Klatte and B. Kummer, Nonsmooth equations in optimizations: Reqularity, calculus, methods and applications, Kluwer academic publ., Dordrecht-Boston, London, 2002.

[34] B.S. Mordukhovich, Sensitivity analysis in nonsmooth optimization: Theoretical Aspects of Industrial Design (D. A. Field and V. Komkov, eds.), SIAM Proc. Appl. Math., 58 (1992), 32-46. 
[35] B.S. Mordukhovich, Variational Analysis and generalized differentiation I: Basic theory, Grundlehren Math. Wiss. 330, Springer-Verlag, Berlin, 2006.

[36] J.P. Penot, Metric regularity, openness and Lipschitzian behavior of multifunctions, Nonlinear Anal., 13 (1989), 629-643.

[37] A. Pietrus, Does Newtons method for set-valued maps converges uniformly in mild differentiability context?, Rev. Colombiana Mat., 34 (2000), 49-56.

[38] A. Pietrus, Non diffrential perturbed Newton's method for functions with values in a cone, Investigation Oper., 35 (2014), 58-67.

[39] L. Qi and J. Sun, A nonsmooth version of Newton's method, Math. Programming A, 58 (1993), 353-367.

[40] M.H. Rashid, On the Convergence of Extended Newton-type Method for Solving Variational Inclusions, Cogent Mathematics, 1:1 (2014), 1-19. DOI:10.1080/23311835.2014.980600.

[41] M.H. Rashid, Convergence Analysis of Extended HummelSeebeck-Type Method for Solving Variational Inclusions, Vietnam J. Math., 44 (2016), 709-726. DOI: 10.1007/s10013-015-0179-2.

[42] M.H. Rashid, Convergence analysis of a variant of Newton-type method for generalized equations, International Journal of Computer mathematics, 95:3 (2018), 584-600. DOI: 10.1080/00207160.2017.1293819.

[43] M.H. Rashid, Extended Newton-type method and its convergence analysis for nonsmooth generalized equations, J. Fixed Point Theory Appl., 19 (2017), 1295-1313 DOI: 10.1007/s11784-0170415-3.

[44] M.H. Rashid, J.H. Wang and C. Li, Convergence Analysis of a Gauss-type Proximal Point Method for Metrically Regualr Mappings, Journal of Nonlinear and Convex Analysis, 14:3 (2013), 627635.

[45] M.H. Rashid and A. Sardar, Convergence of the Newton-type Method for Generalized Equations, GANIT J. Bangladesh Math. Soc., 35 (2015), 27-40.

[46] M.H. Rashid and Y.X. Yuan, Convergence properties of a restricted Newton-type method for generalized equations with metrically regular mappings, Applicable Analysis, in press. DOI:10.1080/00036811.2017.1392018.

[47] M.H. Rashid, S.H. Yu, C. Li and S.Y. Wu, Convergence Analysis of the Gauss-Newton-type Method for Lipschitz-like Mappings, Journal of Optimization Theory and Applications, 158 (2013), 216-233. DOI: 10.1007/s10957-012-0206-3.

[48] S.M. Robinson, Generalized equations and their solutions, part I: basic theory, Math. Progamming Stud., 10 (1979), 128-141.

[49] S.M. Robinson, Newton's method for a class of nonsmooth functions, Set-Valued Analysis, 2 (1994), 291-305.

[50] S.M. Robinson, Generalized equations and their solutions, part II: applications to nonlinear programming, Math. Programming Stud., 19 (1982), 200-221.

[51] R.T. Rockafellar and R.J.-B. Wets, Variational Analysis, Springer-Verlag, Berlin, 1997.

[52] G.N. Silva, Kantorovich's theorem on Newton's method for solving generalized equations under the majorant condition, Appl. Math. Comput., 286 (2016), 178-188.

[53] M. Ulbrich, Semismooth Newton methods for variational inequalities and constrained optimization problems in function spaces, SIAM, Philadelphia, PA, 2011.

[54] X. Xu, Y. Xiao, and T. Liu, Semilocal Convergence Analysis for Inexact Newton Method under Weak Condition, Abstract and Applied Analysis, in press. DOI:10.1155/2012/982925. 